\title{
1 Evolution of the complex transcription network \\ 2 controlling biofilm formation in Candida species
}

3

4 Running title: Evolution of biofilm formation in Candida

5

6 Eugenio Mancera ${ }^{1 *}$, Isabel Nocedal2,3, Stephen Hammel4,5, Megha Gulati6,7,

7 Kaitlin F. Mitchell8,9, David R. Andes ${ }^{8}$, Clarissa J. Nobile ${ }^{6}$, Geraldine Butler4,

8 Alexander D. Johnson².

${ }^{1}$ Departamento de Ingeniería Genética, Centro de Investigación y de Estudios Avanzados del Instituto Politécnico Nacional, Unidad Irapuato, Irapuato, Mexico

${ }^{2}$ Department of Microbiology and Immunology, University of California San Francisco, San Francisco, CA, USA

3Present address - Department of Biology, Massachusetts Institute of Technology, Cambridge, USA

${ }^{4}$ School of Biomolecular and Biomedical Science, Conway Institute, University College Dublin, Dublin 4, Ireland

5Present address - The School of Computer Sciences and IT, Western Gateway Building, University College Cork, Ireland

${ }^{6}$ Department of Molecular and Cell Biology, University of California, Merced, CA, USA

7Present address - Molecular Cell, Cell Press, Cambridge, MA, USA

8Department of Medical Microbiology and Immunology, University of Wisconsin, Madison, United States, USA 
bioRxiv preprint doi: https://doi.org/10.1101/2020.11.08.373514; this version posted November 10, 2020. The copyright holder for this preprint (which was not certified by peer review) is the author/funder. All rights reserved. No reuse allowed without permission.

32

$33 *$ Correspondence: Eugenio Mancera

34 eugenio.mancera@cinvestav.mx

35

36 Keywords: transcriptional evolution, biofilm, Candida albicans, Candida

37 dubliniensis, Candida tropicalis, Candida parapsilosis 
40 We examine how a complex transcription network composed of seven "master"

41 regulators and hundreds of target genes evolved over a span of approximately 70

42 million years. The network controls biofilm formation in several Candida species,

43 a group of fungi that are present in humans both as constituents of the

44 microbiota and as opportunistic pathogens. The ability to form biofilms is crucial

45 for microbial colonization of different host niches, particularly when an

46 implanted medical device is present. We examined and compared the network

47 underlying biofilm formation across four Candida species (C. albicans, $C$.

48 dubliniensis, C. tropicalis, and C. parapsilosis), all of which form biofilms

49 composed of multiple cell types. To describe the salient features of the network

50 across different species, we employed four approaches: (1) we phenotypically

51 characterized the biofilms formed by these species using a variety of methods; (2)

52 we knocked out - one by one - the master regulators identified in C. albicans in

53 the four species and monitored their effect on biofilm formation; (3) we identified

54 the target genes of 18 master regulator orthologs across the four species by

55 performing ChIP-seq experiments; and (4) we carried out transcriptional

56 profiling across each species during biofilm formation. Additional network

57 information was obtained by analyzing an interspecies hybrid formed between

58 the two most closely related species, C. albicans and C. dubliniensis. We observed

59 two major types of changes that have occurred in the biofilm circuit since the four

60 species last shared a common ancestor. Master regulator "substitutions" occurred

61 over relatively long evolutionary times, resulting in different species having 
62 overlapping, but different sets of master regulators of biofilm formation. Second,

63 massive changes in the connections between the master regulators and their

64 target genes occurred over much shorter timescales. Both types of change are

65 crucial to account for the structures of the biofilm networks in extant species. We

66 believe this analysis is the first detailed, empirical description of how a complex

67 transcription network has evolved.

68 


\section{INTRODUCTION}

Many of the most medically relevant fungi belong to the Candida genus.

72 These microbes are part of the human microbiota, but under specific

73 circumstances - such as imbalances in components of the microbiota or

74 suppression of the immune system of the host - they can proliferate as

75 opportunistic pathogens and cause disease (Calderone and Clancy 2012; Turner

76 and Butler 2014; Kullberg and Arendrup 2015; Romo and Kumamoto 2020).

77 These diseases, which were already documented by the ancient Greeks, range

78 from mild cutaneous disorders to systemic infections with high mortality rates

79 (Lynch 1994; Calderone and Clancy 2012; Kullberg and Arendrup 2015; Nobile

80 and Johnson 2015). Although they are usually studied in planktonic (suspension)

81 cultures in the laboratory, Candida species, like many microbes, are often found

82 in nature as biofilms, communities of cells associated with surfaces. For Candida

83 albicans, the best studied and most clinically relevant of the Candida species,

84 biofilms consist of a lower sheet of cells in the yeast form (spherical, budding

85 cells) overlaid by a layer of filamentous cells (hyphae and pseudohyphae) and

86 surrounded by an extracellular matrix composed of proteins and secreted

87 polysaccharides (Blankenship and Mitchell 2006; Nobile and Johnson 2015;

88 Lohse et al. 2018). The matrix, together with specific gene expression changes

89 within biofilms (for example, the upregulation of drug efflux pumps), provide

90 protection from environmental stresses including antifungal drug treatment. The

91 ability of C. albicans to form biofilms has been associated both with its versatility

92 in occupying different niches in the human host and its inherent resistance to 
93 antifungal drugs. These features are especially important for individuals with

94 implanted medical devises, which provide substrates for biofilm formation and

95 where often the only effective treatment is replacement of the device (Donlan

96 2001). Biofilms also shed live yeast-form cells and thereby serve as reservoirs for

97 further colonization in the human body (Nobile and Johnson 2015).

C. albicans biofilm formation begins with the adhesion of yeast cells to a surface, followed by cell division and morphological differentiation to form an

101 upper layer of filamentous cells. The biofilm matures through the secretion of the 102 extracellular matrix (Blankenship and Mitchell 2006; Nobile and Johnson 2015;

103 Lohse et al. 2018). In C. albicans, a complex transcription network regulates this 104 process; it consists of seven "master" transcription regulators (Bcr1, Brg1, Efg1, 105 Flo8, Ndt8o, Rob1 and Tec1) that control each other's expression and, 106 collectively, bind to the control regions of more than a thousand target genes 107 around one sixth of the total number of genes present in the genome of this 108 species (Figure 1) (Nobile et al. 2012; Fox et al. 2015). Despite the complexity of 109 the biofilm regulatory netwrok, several lines of evidence suggest that this network 110 originated relatively recently. For example, genes that are highly expressed 111 during biofilm formation are enriched for genes that are relatively young, 112 meaning that they only have a clear ortholog in species closely related to $C$.

113 albicans (Nobile et al. 2012). Apart from the literature available for C. albicans, 114 most of the work to understand biofilm formation in Candida species has been 115 carried out with Candida parapsilosis (Ding and Butler 2007; Connolly et al. 116 2013; Holland et al. 2014). C. parapsilosis diverged from a last common ancestor 
117 with C. albicans nominally 70 million years ago (Mishra et al. 2007; Butler et al.

118 2009). Although six of the seven master regulators of biofilm formation in $C$.

119 albicans have clear orthologs in C. parapsilosis, only two of them are required for

120 biofilm formation in the latter species (Holland et al. 2014). Candida dubliniensis

121 and C. tropicalis are more closely related to C. albicans (see Figure 2) and are

122 also known to form biofilms (Ramage et al. 2001; Silva et al. 2011; Pujol et al.

123 2015; Araujo et al. 2017; Dominguez et al. 2018; Kumari et al. 2018), but the

124 regulatory circuits that control this process are largely unknown.

125

126 To understand how the complex transcription network that controls

127 biofilm formation evolved, we began with the seven master regulators of biofilm

128 formation in C. albicans and determined whether their orthologs also controlled

129 biofilm formation in $C$. dubliniensis and $C$. tropicalis. Using ChIP-seq, we

130 mapped the targets of the orthologs in C. dubliniensis, C. tropicalis and $C$.

131 parapsilosis. A comparison of the extant networks showed that the two main

132 components of the network, master regulators and target genes, moved in and

133 out of the network at very different rates over evolutionary time. While the

134 regulators moved gradually and in rough correlation with small phenotypic

135 changes in biofilm structure, the master regulator-target gene connections

136 changed very quickly. The large-scale changes in connections observed between

137 closely related species did not appear to have a major impact on biofilm

138 phenotypes, at least as monitored in vitro. These results suggest an evolutionary

139 route through which complex regulatory networks could rapidly explore new 
bioRxiv preprint doi: https://doi.org/10.1101/2020.11.08.373514; this version posted November 10, 2020. The copyright holder for this preprint (which was not certified by peer review) is the author/funder. All rights reserved. No reuse allowed without permission.

140 network configurations (and perhaps new phenotypes) without disrupting

141 existing functions.

142 


\section{RESULTS}

145 Only closely related species to C. albicans form complex 146 biofilms

To understand how the transcription network that controls biofilm

149 formation changed over evolutionary timescale, we first phenotypically

150 characterized the biofilms formed in vitro by the different species of the so-called

151 CTG clade. This clade, which includes but extends beyond Candida species, was

152 traditionally named CTG due to its unusual property of decoding the CTG codon

153 as serine instead of the usual leucine (Figure 2). Recently, this clade has been

154 renamed CTG-Ser1 because other Ascomycota clades were discovered to also

155 have unusual codon usage (Krassowski et al. 2018). To define an optimal growth

156 medium for these assays we tested biofilm formation under several conditions

157 typically used to study biofilms of Candida species (Garcia-Sanchez et al. 2004;

158 Richard et al. 2005; Kucharikova et al. 2011; Nobile et al. 2012; Lohse et al.

159 2017). For the initial tests, we focused on C. albicans and the three species that

160 are most closely related to it and that commonly inhabit humans, C. dubliniensis,

161 C. tropicalis and C. parapsilosis (Figure 2) (Turner and Butler 2014; Gabaldon et

162 al. 2016). The estimated divergence time for $C$. dubliniensis, $C$. tropicalis and $C$.

163 parapsilosis from the last common ancestor with $C$. albicans is approximately

164 20, 45, and 70 million years, respectively (Mishra et al. 2007; Butler et al. 2009;

165 Moran et al. 2012). C. albicans, C. tropicalis and C. parapsilosis have been 
166 previously shown to form biofilms, while less is known about biofilm formation in

167 C. dubliniensis (Silva et al. 2011; Araujo et al. 2017; Dominguez et al. 2018;

168 Kumari et al. 2018). Biofilms were grown in vitro on silicone squares at $37^{\circ} \mathrm{C}$ for

16948 hours with shaking and were monitored by confocal scanning laser

170 microscopy (CSLM), as has been previously described (Nobile et al. 2012). We

171 tested eight different growth media, and only Spider medium with glucose (rather

172 than mannitol) as the carbon source allowed all four species to form thick, well-

173 structured biofilms (Sup Table 1). Our results also showed that environmental

174 conditions are important determinants of biofilm formation for some of these

175 species. While C. albicans formed thick biofilms in all media tested, C. tropicalis

176 biofilm formation, for example, depended very much on carbon source.

Given that there could be differences in the speed at which different

179 species form biofilms, we also assessed biofilm formation as a function of time for

180 the same four species. Biofilms were formed as described above and were 181 monitored at seven different time points from 30 minutes to 96 hours after cell 182 adhesion under the confocal microscope. Although C. albicans formed biofilms 183 more rapidly, by 48 hours all four species had formed mature biofilms that did 184 not significantly change at later time points (Sup Figure 1).

Once we had defined an optimal biofilm growth medium (Spider +

187 glucose) and time point (48 hours) we extended our analysis to other species of 188 the CTG clade (Maguire et al. 2013). In addition to CSLM as described above, we 189 monitored biofilm formation in two additional ways: we determined the biomass 
190 dry-weight of biofilms formed on the bottom of polystyrene plates, and, using a

191 microfluidic flow cell, we continuously monitored biofilm formation by time lapse

192 photography using an optical microscope (Nobile et al. 2012; Lohse et al. 2017).

193 The three methods are complementary: biomass determination is a quantitative

194 method that reduces biofilm formation to a single number, confocal microscopy

195 is qualitative, but allows detailed characterization of the structure of the biofilm,

196 and the microfluidic assay reveals biofilm formation in real-time under a defined

197 flow; the flow rate was adjusted to mimic that of an average catheter implanted in

198 a vein (Gulati et al. 2017; Lohse et al. 2017). Because some of the species we

199 tested are known to grow poorly at $37^{\circ} \mathrm{C}$, we performed the assays at $30^{\circ} \mathrm{C}$ for

200 those species (Kurtzman et al. 2011). Of the 16 species tested, those closest to $C$.

201 albicans formed the thickest biofilms and, in general, the greater the

202 phylogenetic distance from C. albicans the thinner the biofilm formed. Only the

203 two species closest to C. albicans (C. dubliniensis and C. tropicalis) formed

204 biofilms that are structurally very similar to $C$. albicans biofilms, with a basal

205 layer of yeast cells underlying a thick layer of filamentous cells (hyphae and

206 pseudohyphae) enclosed in an extracellular matrix. Biofilms formed by $C$.

207 parapsilosis appeared similar at low resolution, but a closer examination showed

208 that the layer of filamentous cells is composed largely of pseudohyphae rather

209 than a mixture of true hyphae and pseudohyphae. Under these conditions,

210 Lodderomyces elongisporus formed thinner biofilms composed only of yeast

211 cells. Moving further away from C. albicans, Spathaspora passalidarum,

212 Meyerozyma guilliermondii and Clavispora lusitaniae form even thinner

213 biofilms, while Scheffersomyces stipites, Debaryomyces hansenii and C. tenuis, 
214 did not form biofilms under the conditions we tested; only a few cells were

215 observed adhering to the surface. We also performed CSLM assays in an

216 additional medium (RPMI), and the results generally agreed with those described

217 above. Our results with the microfluidic assays showed a similar trend: only those

218 species that are phylogenetically closest to $C$. albicans were able to rapidly form

219 biofilms under flow conditions in the microfluidic device (Sup Figure 2).

222 ascomycetous yeast species that lie outside the CTG clade, C. glabrata and $S$.

223 cerevisiae. Although not closely related to the CTG clade (despite its name), $C$.

224 glabrata is an important opportunistic human pathogen, while $S$. cerevisiae is

225 used extensively in the food and beverage industries and is a widely employed

226 model organism. As can be seen in Figure 2, neither of these species formed

227 biofilms that resembled those formed by C. albicans and its close relatives in the

228 assays and conditions that we tested.

To assess whether the results observed in vitro can be recapitulated in

231 vivo, we tested the ability of $C$. albicans, C. dubliniensis, C. tropicalis and $C$.

232 parapsilosis to form biofilms in the rat catheter model, a well-established in vivo

233 biofilm model (Andes et al. 2004). All four species were able to form biofilms,

234 although the biofilms formed by $C$. albicans and $C$. dubliniensis were

235 considerably thicker and more filamentous (Figure 2C). These results agree with

236 previous in vivo characterizations performed for C. albicans and C. parapsilosis 
237 (Nobile et al. 2012; Connolly et al. 2013) and provide new information on the

238 intermediate species.

240 In summary, our results show that the ability to form biofilms that

241 resemble those of $C$. albicans is limited to its most closely related species. In

242 terms of biomass, there is a sharp drop off outside C. parapsilosis while, in terms

243 of biofilm structure, only $C$. dubliniensis and C. tropicalis form biofilms similar

244 to those of $C$. albicans, in terms of all three morphological cell types being

245 represented. Of all the species studied, $C$. albicans biofilm formation is the most

246 rapid and most robust to environmental changes; moreover, the biofilms formed

247 by this species are the most stable to physical manipulation (results not shown).

250 The regulatory core of the biofilm transcription network 251 changed gradually over time

To gain insight into the evolutionary changes that occurred in the

254 transcription network that controls biofilm formation at a molecular level, we 255 first studied the function of the seven master regulators of the C. albicans 256 network (Figure 1). Given the phenotypic results described above, we centered

257 the analysis on C. albicans, C. dubliniensis, C. tropicalis and C. parapsilosis. All 258 four of these species are common in humans (Turner and Butler 2014), and the 259 first three form similar structural types of biofilms. As described above, $C$. 260 parapsilosis also forms biofilms, but its biofilms show more pronounced 
261 differences. All seven master regulators of the network in C. albicans (Figure 1)

262 have clear orthologs in the other three closely related species, with the exception

263 of Rob1. Rob1 has a patchy phylogenetic distribution, with syntenic orthologs

264 present in C. albicans, C. dubiniensis and C. tropicalis, but apparently absent

265 from C. parapsilosis and closely related species. However, Rob1 orthologs are

266 present in other more distantly related CTG species, which supports the

267 hypothesis that Rob1 was either lost or was evolving sufficiently rapidly in the $C$.

268 parapsilosis linage that it cannot be recognized (Maguire et al. 2013).

270 To test whether the orthologs of the C. albicans master regulators are

271 involved in biofilm formation in the other species, we generated gene deletion

272 knockouts in C. dubliniensis and C. tropicalis. The knockouts in C. albicans and

273 C. parapsilosis had been previously generated as part of large transcription

274 regulator deletion projects (Homann et al. 2009; Holland et al. 2014). To make

275 the knockouts in $C$. dubliniensis and $C$. tropicalis we used amino acid 276 auxotrophic strains and employed a gene knockout strategy similar to the that

277 previously used for C. albicans and C.parapsilosis (Mancera et al. 2019).

The ability of the different gene knockout strains to form biofilms was

280 monitored by biomass dry-weight determination and by CSLM. As can be 281 observed in Figure 3 and Sup Fig. 3, all seven master regulators identified in $C$.

282 albicans were also required for biofilm formation in C. dubliniensis. The results 283 were different for C. tropicalis; here, only five of the seven were required, with 284 Rob1 and Flo8 appearing dispensable for biofilm formation under our laboratory 
285 conditions. The biofilms formed by the ROB1 and FLO8 deletion mutants in $C$.

286 tropicalis were actually slightly heavier and the hyphal layer was denser than

287 biofilms formed by the parental (wildtype) strain, suggesting that these two

288 regulators may have assumed a subtle inhibitory role in these species. For $C$.

289 parapsilosis, it was previously shown that, of the seven master biofilm regulators

290 in C. albicans, only Bcr1 and Efg1 are indispensable for biofilm formation. The

291 Ntd8o deletion in C. parapsilosis fails to form biofilms but also has a general

292 growth defect, making it difficult to ascertain its precise role. Previous work also

293 established that deletion of $C_{2 F}, U M E 6, C P H_{2}, G Z F_{3}$ and $A C E 2$ all exhibit

294 biofilm specific defects in C. parapsilosis but not in C. albicans (Holland et al.

295 2014). Overall, our results, together with previous observations, show that the

296 group of master regulators underlying biofilm formation in C. albicans has

297 diversified in other Candida species with the degree of diversity roughly

298 paralleling their evolutionary distance from C. albicans.

301 Target genes of the master biofilm regulators differ greatly among Candida species

To determine how the binding connections between the biofilm master 305 regulators and their target genes have changed over evolutionary time, we 306 determined genome-wide protein-DNA interactions of the master regulators in $C$.

307 albicans, C. dubliniensis, C. tropicalis and $C$. parapsilosis by chromatin 308 immunoprecipitation followed by next-generation sequencing (ChIP-seq) 
309 (Johnson et al. 2007). To this end we tagged each of the regulators with a Myc

310 epitope tag that can be immunoprecipitated using a commercially available

311 antibody. This strategy has the advantage that a single antibody with the same

312 affinity could be used in all experiments, and control experiments could be

313 performed with untagged strains. All the ChIP-seq experiments were performed

314 in mature biofilms grown for 48 hours at $37^{\circ} \mathrm{C}$ in the optimal medium described

315 above. For unknown technical reasons, not all regulators could be

316 immunoprecipitated in all species. Of all the ChIP-seq experiments performed,

317 protein-DNA interactions could be reliably determined for 18 regulators across

318 the four species (Sup Table 2). We believe that this coverage, although not

319 complete, is more than sufficient to uncover the general trends of biofilm network

320 evolution.

321

The large amount of new genome-wide protein-DNA interaction data

323 reported here will be useful in future studies of biofilm formation in these

324 Candida species. Very few "structural" genes have been implicated in biofilm

325 formation, and the ChIP-seq results across species could greatly facilitate the

326 identification of key non-regulatory genes required for biofilm formation. For

327 example, specific master regulator-target gene connections that are preserved

328 across multiple species may point to target genes that are especially important for

329 biofilm formation. Such a hypothesis could be tested by knocking out the target

330 gene of interest. In the following sections, we consider, in general terms, how the

331 biofilm network (defined as the set of master regulators and connections between 
332 them and their target genes) differs among Candida species and what these

333 differences reveal about the evolution of the network.

To compare gene targets between species, we assigned each ChIP 336 occupancy site to the ORF with the nearest downstream start codon. To compare

337 the changes in master regulator-target gene connections across species, were first 338 examined the target genes that are conserved across the species. The overall 339 percentage of one-to-one orthologs between the four species ranged from a high 340 of $91 \%$ between $C$. albicans and $C$. dubliniensis to a low of $74 \%$ between $C$.

341 tropicalis and C. parapsilosis (Maguire et al. 2013). If we consider only the one-

342 to-one orthologs, it becomes clear that the connections between master

343 regulators and conserved target genes vary greatly across these species (Figure 4).

344 Between the two most closely related species (C. albicans and $C$. dubliniensis),

345 the master regulator that showed the highest conservation of target gene 346 connections was Ndt8o, but the overlap was only about 50\%. Between C. albicans

347 and $C$. parapsilosis, this value drops to about $25 \%$. Although only $12 \%$ of Ndt8o

348 target gene connections are common to all four species, the overlap for each 349 species pair is larger than expected by chance (hypergeometric test, $P<0.05$ ), 350 indicating that a small but significant group of Ntd80-target gene connections

351 have been preserved across these species. The other master regulators show an 352 even lower degree of conserved regulator-target gene connections. For example, 353 Rob1 shares only about $12 \%$ of its target gene connections between the two most 354 related species, C. albicans and C. dubliniensis. 
Our results also show that each species has target genes in its biofilm

357 network that lack orthologs in the other three species. In C. albicans, $26 \%$ of the

358 genes bound by at least one of the biofilm master regulators do not have

359 orthologs in the other three species, compared with $19 \%$ for the genome as a

360 whole. This analysis extends the previous observation that genes that are

361 overexpressed during biofilm formation in C. albicans are often "young" genes,

362 that is genes that lack orthologs in related species (Nobile et al. 2012). We

363 observed a similar enrichment of unique genes in the biofilm network of $C$.

364 parapsilosis, but not for $C$. dubliniensis and $C$. tropicalis; in the latter two

365 species the fraction of non-orthologous genes in the biofilm network was

366 approximately the same as that observed for the whole genome. These

367 observations indicate that the biofilm networks of C. albicans and C. parapsilosis

368 have been more dynamic in recent evolutionary time than those of the other two 369 species.

376 albicans showed that many target genes were directly connected (by binding) to

377 more than one master regulator (Nobile et al. 2012), and this general feature of

378 the network architecture is observed across the Candida species studied here,

379 despite the low conservation of individual regulator-target gene connections 
380 (Figure 4). Perhaps the most notable example is seen by comparing the target 381 genes of Ndt8o and Efg1 across the four species. The set of target genes of Ndt8o

382 is considerably larger, but over $75 \%$ of the Efg1 target genes are also Ndt8o

383 targets in all four species. In addition, the binding motifs of these two regulators

384 are enriched in each other's binding locations in all four species studied (Figure

385 5A). These observations indicate that, in all species, Efg1 binds in conjunction

386 with Ndt80 even though the target genes of the regulator combination differ

387 greatly across species. The association between Efg1 and Ndt8o agrees with

388 previous planktonic ChIP-seq experiments of Efg1 performed in C. parapsilosis

389 where the most enriched binding motif found was that of Ndt8o (Connolly et al.

390 2013). Taken together, these results suggest that the Efg1-Ndt8o association is

391 ancient with respect to the Candida species studied here and that it remains

392 preserved across them despite large species to species differences in the target

393 genes bound by the two regulators. Analysis of the other master regulators

394 indicates that combinational control of target genes is very common in all species, 395 although the other examples do not seem as deeply conserved as the Efg1-Ndt8o 396 example.

In terms of overall network structure across species, we also examined

399 whether, as is the case for C. albicans, the master regulators bind to their own 400 control region as well as those of the other master regulators. The extent of 401 conservation of the binding connections between one master regulator and the 402 others varies from regulator to regulator (Sup Table 4), with Ndt8o showing the 403 highest conservation. In all four species, Ndt8o binds to its own control region as 
404 well as those of all six other regulators, with the exception of the control region of

405 FLO8 in C. albicans and ROB1 in C. dubliniensis. Efg1, Brg1 and Tec1, in that

406 order, follow Ndt8o in their degree of connection conservation, with Rob1 and

407 Bcr1 having the least conserved set of connections. However, we note that the

408 binding data for these two regulators is also the least complete. Ndt8o and Efg1

409 each bind to their own control regions in all the four species analyzed, while Brg1

410 exhibits this interaction only in C. albicans and C. dubliniensis. The binding of

411 the other regulators to their own upstream intergenic regions appears less

412 conserved. Although the ChIP data is unlikely to capture all relevant interactions,

413 we can analyze the existing data to determine whether the connections between

414 regulators (including a regulator binding to its own control region) are more

415 prevalent than that expected by chance. We randomly choose 2,000 groups of

416 seven genes, the number of master regulators in the network, from the binding

417 sets in each species and counted the total number of interactions with the master

418 regulators. In all four species, the number of connections observed between the

419 regulators is greater than what would be expected by chance $(P<<0.05)$. Overall,

420 our findings show that high connectivity between the master regulators is

421 conserved in the four Candida species analyzed. Thus, despite the extensive

422 changes in the network across species, the high connectivity among regulators

423 remains a structural feature of the network in each species. 
We considered the possibility that our results could be skewed by false

430 positive signals intrinsic to ChIP-seq experiments, even when proper controls are

431 used (Chen et al. 2012). To deal with this potential problem, we employed two

432 additional criteria for increasing stringency in our analysis of transcription

433 networks (Nocedal et al. 2017). First, we filtered the regions identified as

434 enriched in ChIP signal to include only those regions that also had a high scoring

435 regulator binding motif in the intergenic region. As discussed below, the binding

436 motif for some of the master regulators does not vary significantly across the

437 species we studied. Second, we also incorporated gene expression data to further

438 filter the gene targets to those that, (1) have ChIP enrichment, (2) show the

439 presence of a regulator binding motif in the intergenic region, and (3) whose

440 expression changes under biofilm forming conditions. Although the gross

441 number of regulator-target gene connections decreased as the stringency of the

442 filtering criteria increased, the high proportion of differences across Candida

443 species described above did not significantly change (Sup Figure 4).

445 Another potential caveat which could confound our analysis concerns our 446 conclusions being based on the specific conditions under which we induced

447 biofilm formation. To test whether this concern is significant, we performed the 448 ChIP-seq experiments for Ndt8o in C. albicans, C. dubliniensis and C. tropicalis 449 in biofilms grown in an alternative growth media. Although, in all species, the 450 regulator-target gene connections differed between the two media (Sup Figure 5), 
451 the magnitude of the differences in regulator-target gene connections across

452 species was similar to that of our original analysis.

455 Changes in master regulator-target gene interactions are due 456 to changes in cis-regulatory regions

460 in the trans components of the network, for example, changes in the DNA-

461 binding specificity of a master regulator. This type of change has the potential to

462 dramatically change the network over relatively short evolutionary time scales. To

463 explore this possibility, we examined the ChIP-seq binding data for motifs

464 recognized by the master regulators. Performing de novo motif searches, we

465 found that the enriched DNA-binding motifs found in the binding regions of the

466 biofilm master regulators were very similar across the four species we examined

467 (Figure 5). Moreover, the de novo generated DNA-binding motifs for Efg1 and

468 Ndt8o are similar to those previously reported for their orthologs in other species

469 (Nobile et al. 2012; Nocedal et al. 2017). Overall, this analysis shows that the

470 DNA-binding specificity of at least some of the master regulators has not changed

471 significantly across these species and cannot explain the diversity of master

472 regulator-target gene connections observed across the species. This conclusion is

473 further supported by analysis of a C. albicans-C. dubliniensis hybrid, as described 474 below. 
477 Biofilm specific gene expression changed rapidly over 478 evolutionary time master regulator-target gene connections documented above is that the genes

482 transcriptionally induced during biofilm formation should differ substantially 483 among species. To test this prediction, we generated genome-wide transcription

484 profiles of $C$. albicans, C. dubliniensis and C. tropicalis under biofilm forming 485 conditions. To reveal biofilm-specific changes, we compared these profiles to 486 expression data obtained when the species were grown in suspension cultures in 487 the same medium. As a reference, we also performed the biofilm expression 488 profile in a second C. albicans isolate. As seen in Figure $5 \mathrm{~B}$, the pairwise 489 differences in the transcription profiles across the three species are significant 490 and reflect their phylogenetic position: the further apart the two species are from 491 one another, the less correlated their transcription profiles are. If we use a lax 492 cut-off of two-fold over-/under-expression to define genes that change their 493 expression during biofilm formation, the overlap between pairs of species is 494 relatively low. For example, only $29 \%$ of the genes that change their expression 495 during biofilm formation are shared between C. albicans and C. dubliniensis, and 496 only $24 \%$ are shared between $C$. albicans and C. tropicalis. The overlap of 497 differentially expressed genes between the two C. albicans isolates was $48 \%$. This 498 result is consistent with previous work showing that clinical isolates differ in their 
499 biofilm forming abilities (Hirakawa et al. 2014; Huang et al. 2019). As noted in

500 the discussion, we believe that many of the differences among clinical strains

501 arose after the C. albicans-C. dubliniensis branch.

502

503 To test whether the large differences in the gene expression profiles were 504 specific to the media conditions used, we also performed the transcription 505 profiles in different media, namely Spider for C. albicans and C. dubliniensis, and 506 RPMI for C. albicans and C. tropicalis. In the alternative media, the conservation 507 between the sets of genes that changed their expression at least twofold during 508 biofilm formation are even lower, $22 \%$ and $11 \%$, respectively for C. albicans and 509 C. dubliniensis, and C. albicans and C. tropicalis. Despite these major 510 differences, all the interspecific pairwise overlaps are greater than would be 511 expected by chance (hypergeometric test, $P<<0.5$ ). Overall, the low degree of 512 conservation in genome-wide gene expression, agrees well with the low 513 conservation of regulator-target gene connections across species described above.

Analysis of an interspecies hybrid independently supports

517 the conclusions from the species-to-species comparisons

520 connections in transcription networks between yeast species and, more generally,

521 between any species (Chen et al. 2012). These difficulties include technical issues

522 such as differential nucleic acid recovery and signal to noise ratios, which can 
523 vary considerably from one species to the next. However, probably the most

524 difficult problem to circumvent arises from different species having different

525 physiological responses to the same external environment. For example, $30^{\circ} \mathrm{C}$

526 could be the optimal temperature for one species but might induce a stress

527 response in a closely related species. Therefore, a network comparison between

528 these species at $30^{\circ} \mathrm{C}$ might be dominated, not by evolutionary changes in the

529 transcription circuitry per se, but simply by the fact that only one species has

530 induced a stress response. This problem can be overcome by creating and

531 analysing interspecies hybrids, where the genomes of two different species are

532 present in the same cell and thus exposed to the same physiological state (Wilson

533 et al. 2008; Nocedal et al. 2017). This approach, which can only be carried out

534 between closely related species, specifically reveals the cis-regulatory changes

535 that have accumulated between the two genomes since the species last shared a

536 common ancestor.

We took advantage of the fact that it is possible to mate $C$. albicans and $C$.

539 dubliniensis (each diploid) to generate tetraploid hybrids (Pujol et al. 2004).

540 These hybrids form biofilms similar to those formed by C. albicans (Sup Fig 6).

541 We performed ChIP-seq of Ndt8o in this hybrid, immunoprecipitating the $C$.

542 albicans Ndt8o protein in one set of experiments and the C. dubliniensis Ndt8o

543 in another set. The results showed that - in the hybrid - the target genes bound

544 by the $C$. albicans Ndt8o and the $C$. dubliniensis Ndt8o were highly correlated,

545 similar to two biological replicates carried out in the same species (Figure 6A). In

546 other words, we obtained the same target genes in the hybrid regardless of which 
547 Ndt8o was tagged for immunoprecipitation. Importantly, the binding positions

548 on the $C$. dubliniensis genome in the hybrid were characteristic of the results in

549 C. dubliniensis, specifically $97 \%$ of the targets in the hybrid are targets in $C$.

550 dubliniensis, and the positions on the C. albicans genome were characteristic of

551 C. albicans with $96 \%$ of the targets in the hybrid being targets in C. albicans.

552 Although we only carried out this experiment with one master regulator, the

553 results independently validate our earlier conclusions based on the much more

554 extensive species-to-species comparisons. These observations confirm our

555 previous conclusion that the extreme differences in regulator-target gene

556 connections observed across Candida species are due to changes in the cis-

557 regulatory sequences in the target genes rather than changes in the regulators

558 themselves or differences in the physiological state of the species at the time of

559 analysis. 


\section{DISCUSSION}

In this paper, we examined how a complex transcriptional network 563 underlying a specific phenotype (Figure 1) evolved over a span of approximately

56470 million years. The phenotype is biofilm formation by Candida species, a group 565 of fungi that colonize humans, sometimes leading to disease. We documented 566 phenotypic differences in biofilm formation across many fungal species, and we

567 mapped the transcriptional networks underlying biofilm formation in four of

568 them, C. albicans, C. dubliniensis, C. tropicalis and C. parapsilosis. All four 569 species form complex biofilms both in vitro and in vivo in a rat catheter model 570 (Figure 2).

571 Using C. albicans as a reference species, our analysis leads to the following

573 five main conclusions. (1) as we move away from C. albicans, biofilms become 574 less complex, both in terms of structure and of composition; that is, fewer cell 575 types are involved and the resulting biofilm is less regular. At larger evolutionary 576 distances, fungal species did not form biofilms at all under the conditions we 577 tested. (2) Of the seven master transcriptional regulators of biofilm formation in 578 C. albicans, all seven are needed in the most closely related species $(C$. 579 dubliniensis), but, as we move further away evolutionarily, fewer are required for 580 biofilm formation. For example, two of the master regulators (Rob1 and Flo8) are 581 dispensable for biofilm formation in the next most closely related species $(C$. 582 tropicalis), and three of the seven are not required in C. parapsilosis. As shown 583 by Holland and colleagues (Holland et al. 2014), other transcriptional regulators 
584 (present in C. albicans but not required for biofilm formation in this species)

585 have assumed the role of master regulators in C. parapsilosis. (3) In contrast to 586 the relatively slow evolutionary substitutions of master regulators, the

587 connections between the master regulators and their target genes have changed 588 very rapidly over evolutionary time (Figure 6B). This conclusion is most obvious 589 when we compare the two most closely related species, $C$. albicans and $C$. 590 dubliniensis, estimated to have last shared a common ancestor 20 million years 591 ago. Depending on the regulator, fewer than 50\% of the master regulator-target 592 gene connections were observed to be conserved. This conclusion was 593 independently verified for one regulator - Ndt8o - by analyzing its binding 594 distribution across the two genomes in a C. albicans-C. dubliniensis hybrid; here, 595 the binding distribution of Ndt8o across one genome differed considerably from 596 that of the other, and each resembled that seen in the cognate individual species. 597 This result strongly supports the conclusion that the differences in regulator598 target gene connections across species are due largely to changes in the cis599 regulatory sequences of the target genes rather than changes in the regulators. (4) 600 As predicted from the extensive changes in regulator-target gene connections, 601 mRNA expression during biofilm formation differs considerably from one species 602 to the next. Like the other changes we have documented in this paper, mRNA 603 expression divergence becomes greater as the phylogenetic distance increases 604 (Figure 6). (5) Despite the extensive changes in the transcription networks 605 underlying biofilm formation across the species we examined, several key 606 features of the overall architecture of the network appear to be preserved. For 607 example, all species show high connectivity in the sense that many target genes 
608 are directly connected (by binding) to more than one master regulator. Moreover,

609 many of the master regulators bind to their own control regions as well as those

610 of the other master regulators. We have argued elsewhere that these two features

611 are likely to be common to many complex transcription networks (Sorrells and

612 Johnson 2015), and the results presented here show that, despite many changes

613 in individual regulator-target connections, the basic "structural features" of the

614 network are preserved across the biofilm networks of the four species examined.

To place our findings in context it is also instructive to compare our

617 analyses of biofilm formation across species with recent studies where biofilm

618 formation has been analyzed across different isolates of a single species, $C$.

619 albicans (Hirakawa et al. 2014; Huang et al. 2019). Hirakawa et al. (2014)

620 determined the genome sequences of 21 clinical isolates of $C$. albicans and

621 examined their abilities to form biofilms. The genome comparisons revealed

622 many differences among strains including aneuploidies, losses of heterozygosity,

623 and mutations in coding sequences; moreover, the strains differed substantially

624 in their abilities to form biofilms. Among the strains analyzed, the one used in

625 our study (SC5314) was among the thickest biofilm producers as assayed by dry

626 weight; the majority of isolates formed thinner biofilms. One clinical isolate that

627 formed very poor biofilms was found to have an inactivating mutation in EFG1,

628 one of the biofilm master regulators, indicating a relatively recent change.

629 Because the $C$. dubliniensis strain used in our study (CD36) formed biofilms that

630 are similar to those of strain SC5314, we believe that $\mathrm{SC} 5314$ is a good

631 representative of $C$. albicans and that most of the clinical isolates probably 
632 acquired mutations (including aneuploidies and losses of heterozygosity)

633 relatively recently. Huang et al. (2019) examined five of the previously sequenced

634 strains in much more detail including the dependence on individual

635 transcriptional regulators for biofilm formation. Although the magnitude of the

636 effect of transcriptional regulator deletions on biofilm formation varied across

637 strains, SC5314 again appears to be a good representation of the ability of $C$.

638 albicans as a species to form thick, complex biofilms.

640 To our knowledge, this study is the first to examine in detail how a 641 complex transcription network changes over a relatively short evolutionary time

$642-70$ million years - represented by four different species. During that time, the

643 master transcription regulators controlling biofilm formation have slowly 644 changed, but their connections to the target genes they control have changed 645 rapidly. We do not know which, if any, of these changes were adaptive; in this 646 regard, it is important to note that, although the biofilms produced by the

647 Candida species have many similarities, they do differ from species to species in

648 at least subtle aspects. Even considering the two most closely related species $(C$.

649 albicans and C. dubliniensis), it is possible to distinguish their biofilms. Although

650 they appear very similar under the confocal microscope, the C. albicans biofilms

651 form faster and under a greater range of conditions; once formed, they are more

652 difficult to disrupt than those of C. dubliniensis. Although these differences may

653 help to explain why $C$. albicans is a greater problem in the clinic than $C$.

654 dubliniensis, it is difficult to reconcile these subtle differences in phenotype with

655 the large differences in the underlying transcriptional circuitry. Given the large 
656 magnitude of changes underlying such similar phenotypic output, we propose as

657 a default hypothesis that many of the changes in transcription circuitry result

658 from neutral evolution, more specifically, constructive neutral evolution whereby

659 molecular complexity can change without an increase in fitness (Stoltzfus 1999;

660 Lynch 2007; Wagner 2014; Sorrells and Johnson 2015; Brunet and Doolittle

661 2018). This study clearly shows that complex transcription networks responsible

662 for the same basic phenotype can undergo evolutionary changes that appear

663 much greater in magnitude than the resulting differences in phenotype.

664

665 
METHODS

\section{Characterization of biofilm formation}

670 the different species and strains was performed on silicon squares as described

671 previously (Nobile et al. 2012). The strains used for each species and media

672 employed are shown in Sup Table 4 and 1, respectively. Briefly, for the adhesion

673 phase, silicone squares pre-treated with adult bovine serum albumin (BSA) were

674 inoculated to an $\mathrm{OD}_{600}$ of 0.5 with cells from an overnight culture grown at $30^{\circ} \mathrm{C}$

675 in YPD medium. After incubation for 90 minutes at $37^{\circ} \mathrm{C}$ and $200 \mathrm{rpm}$ in the

676 specific medium (Sup Table 1) for adhesion, the squares were washed with

677 phosphate-buffered saline (PBS) and then placed in fresh media and incubated

678 for 48 hours at $37^{\circ} \mathrm{C}$ and $200 \mathrm{rpm}$. Biofilms of the species that do not grow well at $67937^{\circ} \mathrm{C}$ were grown at $30^{\circ} \mathrm{C}$ as indicated in Figure 2. After 48 hours, the biofilms

680 were stained for $1 \mathrm{hr}$ with $50 \mathrm{mg} / \mathrm{mL}$ of concanavalin A-Alexa Fluor 594 681 conjugate and visualized on a Nikon Eclipse C1si upright spectral imaging 682 confocal microscope using a 40x/o.8oW Nikon objective.

684 Visualization of biofilm formation over time was also performed by CSLM in 685 Spider medium. For each of the four species observed (C. albicans, $C$. 686 dubliniensis, C. tropicalis and C. parapsilosis), seven independent silicone 687 squares were used for biofilm formation as described above and the biofilms at 688 each square were visualized at 30 minutes, 4, 8, 12, 24, 48 and 96 hours after the 689 adhesion phase (Sup Figure 1). 
691 Determination of the biomass dry-weight of biofilms of the different 692 species and strains was performed by growing biofilms on the bottoms of 6-well 693 polystyrene plates pre-treated with BSA as previously described (Nobile et al.

694 2012). The silicone squares were adhered for 90 minutes. These assays were 695 performed in a modified Spider medium that contained 1\% glucose rather than 696 mannitol as the carbon source. After 48 hours of biofilm formation, supernatants 697 were aspirated and biofilms were scraped and placed to dry on top of a filter 698 paper. Dried biofilms were weighed on an analytical scale subtracting the weight 699 of a filter paper in which the media without cells was filtered. Five technical 700 replicates were performed per strain. As was performed for CSLM visualization, 701 strains that do not grow well at $37^{\circ} \mathrm{C}$ were grown at $30^{\circ} \mathrm{C}$.

702

Biofilm formation in a Bioflux microfluidic device (Fluxion Biosciences)

704 was assayed as described previously (Gulati et al. 2017). The medium used was 705 Spider with $1 \%$ glucose and without mannitol, and assays were performed at $37^{\circ} \mathrm{C}$ 706 and $30^{\circ} \mathrm{C}$ for 24 hours.

707

708 In vivo biofilm formation assays were performed using the rat central709 venous catheter infection model as previously described (Andes et al. 2004). 710 After 24 hours of infection by the four species tested, biofilm formation on the 711 intraluminal surface of the catheters was observed by scanning electron 712 microscopy (SEM). Procedures were approved by the Institutional Animal Care 
713 and Use Committee (IACUC) at the University of Wisconsin, Madison (protocol

714 MV1947).

715

716 Generation of gene deletion knockout strains

717 Gene deletion knockout strains were constructed using a similar fusion

718 PCR strategy as that described by Noble and Johnson (2005) employing histidine

719 and leucine auxotrophic strains. Construction of these strains was performed

720 using the $S A T 1$ flipper strategy as previously described (Mancera et al. 2019). All

721 the strains employed and generated in this study are shown in Sup Table 4. In

722 brief, the two alleles of each regulator in C. dubliniensis were subsequently

723 deleted using the C. albicans HIS1 and LEU2 genes. In C. tropicalis the first allele

724 was deleted using the C. albicans LEU2 gene while the second was deleted using

725 the $C a H y g B$ gene that confers resistance to hygromycin B (Basso et al. 2010). To

726 generate the gene-deletion cassettes, $\sim 350$ bp flanking 5' and 3' regions of each

727 regulator where PCR amplified from genomic DNA, and fused to the

728 corresponding auxotrophic/drug resistance marker by fusion PCR.

729 Transformation was performed by electroporation as previously described

730 (Porman et al. 2011). Verification of correct integration of the gene deletion

731 cassettes was performed by colony PCR with primers directed to both flanks of

732 the disrupted gene. Final gene-deletion confirmation was performed by colony

733 PCR with primers that anneal at the ORF of each regulator. Two independent

734 isolates of each deletion mutant originating from two separate transformations

735 were generated for each regulator deletion. The regulator knockout strains of $C$.

736 albicans and C. parapsilosis had been previously generated as part of efforts to 
737 generate collections of regulator gene knockout strains (Homann et al. 2009;

738 Holland et al. 2014).

744 identify the target genes of the seven regulators was performed as previously

745 described (Hernday et al. 2010; Lohse and Johnson 2016), and sequenced using

746 Illumina HiSeq 2500 or 4000 platforms. Each of the seven regulators in $C$.

747 dubliniensis and C. tropicalis was tagged in the wildtype strain background with

748 a 13x Myc epitope tag at the C-terminus from the pADH34 or pEMo19 plasmids,

749 respectively, as previously described (Hernday et al. 2010; Mancera et al. 2019).

750 C. albicans Myc-tagged strains had been similarly generated previously (Nobile et

751 al. 2012). C. parapsilosis Brg1, Ndt8o and Tec1 were tagged using a 6x C-

752 terminal Myc tag amplified from plasmid pFA-MYC-HIS1 as described by

753 (Connolly et al. 2013). C. parapsilosis Efg1 had been previously tagged (Connolly

754 et al. 2013). We were not successful tagging Bcr1 in this species. Genotype details

755 for all the strains generated and used are in Sup Table 4.

757 To generate the C. albicans/C. dubliniensis tagged hybrid strains, the a or

758 a allele of the mating-type-like (MTL) locus was deleted in the Ndt8o tagged

759 strains described above. These deletions allowed the strains to become capable of

760 white-opaque switching, and thus mating competent. In C. albicans, the $\alpha$ allele 
761 of the MTL locus was deleted by replacing it with $A R G 4$ using the plasmid pJD1

762 as previously described (Lohse et al. 2016). In C. dubliniensis, the a allele of the

763 MTL locus was deleted using a cassette containing the SAT1 nourseothricin

764 resistance marker from plasmid pSFS2A flanked by $\sim 300$ bp homology regions

765 identical to the 3' and 5' upstream/downstream regions of the $M T L$ locus.

766 pSFS2A is a plasmid derived from pSFS2 (Reuss et al. 2004) that contains the

$767 S A T 1$ reusable cassette in the backbone of vector pBC SK+ instead of pBluescript

768 II KS and that was kindly provided by Joachim Morschhauser (U. Würzburg).

769 The deletion of the $\alpha$ or a alleles was verified by colony PCR of the two flanks.

770 Generation of the hybrid strains was done by overlaying the mating competent

771 wildtype and Myc-tagged strains on a YPD plate for 48 hours at $30^{\circ} \mathrm{C}$. Single

772 colonies were then streaked out and hybrids were selected by growing on media

773 containing nourseothricin and lacking arginine. The hybrid strains were further

774 verified measuring DNA content using FACS. As controls, the two wildtype

775 untagged mating competent strains were hybridized.

776

777 All immunoprecipitation experiments were performed under biofilm 778 growth conditions in 6-well polystyrene plates as previously described (Nobile et

779 al. 2012). After 48 hours of biofilm growth in Spider $1 \%$ glucose at $37^{\circ} \mathrm{C}$ and 200

$780 \mathrm{rpm}$, cells were fixed with $1 \%$ formaldehyde for 15 minutes. Cell disruption and

781 immunoprecipitation were performed as previously described (Hernday et al.

782 2010) using a c-Myc tag monoclonal antibody (RRID: AB_2536303). After

783 crosslink reversal, instead of performing a phenol/chloroform extraction, we

784 used a MiniElute QIAGEN kit to purify the immunoprecipitated DNA. Library 
785 preparation for Illumina sequencing was performed using an NEBNext ChIP-Seq

786 Library Prep Master Mix Set for Illumina sequencing. Between 12 and 24 samples

787 were multiplexed per lane. As controls, immunoprecipitations were performed in

788 matched strains that lacked the Myc tag. Two biological replicates were 789 performed for each regulator in the four species.

792 Identification of regulator directly bound target genes by ChIP793 seq

ChIP-seq reads were mapped to their corresponding genome using Bowtie

7952 with default parameters (Langmead and Salzberg 2012). The genome sequences

796 and annotations were obtained from CGD for versions: 06-Apr-

797 2014C_albicans_SC5314_A21, o6-Nov-2013C_dubliniensis_CD36, 11-Dec-

798 2013C_tropicalis_MYA-3404, and C_parapsilosis_CDC317_version_so1-mo3-

799 r13. The SAMtools package was used to convert, sort and index the sequenced

800 reads to $\mathrm{BAM}$ format ( $\mathrm{Li}$ et al. 2009). We observed that the peak calling

801 algorithm was more specific and sensitive if the number of reads in the treatment

802 and control datasets were similar. Therefore, we adjusted the number of reads in

803 the different treatment-control dataset pairs using SAMtools view -s function

804 prior to peak calling. Peak calling was performed using MACS2 (Zhang et al.

805 2008) with a q-value cutoff of 0.01; the shiftsize parameter was determined using

806 the SPP package in R (Kharchenko et al. 2008). Peaks were considered as true

807 binding events only if the peak was identified in both biological replicates.

808 Assignment of peaks to ORFs was done using MochiView when the peak was 
809 present in the intergenic region immediately upstream of the ORF (Homann and

810 Johnson 2010).

811

812 To identify regulator binding target genes in the hybrid strains, ChIP-seq

813 reads were aligned to the $C$. albicans and $C$. dubliniensis genomes as described

814 above. Reads that aligned to both genomes were subsequently filtered out.

815 Further processing, peak calling, and assignment of peaks to ORFs were then 816 performed independently for reads that mapped to the $C$. albicans and $C$.

817 dubliniensis genomes as described above.

De novo sequence motif discovery and enrichment for the

821 regulators

DNA-binding motifs were generated de novo for regulators from ChIP-Seq

823 experiments using DREME (Bailey 2011). The union of the sequences under the

824 peaks of the two biological replicates for each experiment were tested against a

825 background of matched length random genomic sequences from that species. The

826 top scoring motif was taken and is shown in Figure 5.

827

828 Based on the motifs generated de novo using DREME as well as previously

829 reported DNA-binding motifs (Lassak et al. 2011; Nobile et al. 2012; Connolly et

830 al. 2013; Nocedal et al. 2017), a high-confidence "consensus motif” was generated

831 for Ndt8o (CACAAA) and Efg1 (TGCAT). To determine enrichment of these

832 consensus motifs in peaks identified for each ChIP-Seq experiment, the number 
833 of consensus motifs in the union of the sequence under the peaks of both

834 biological replicates was compared to the number of motifs in intergenic regions

835 for that species. A Fisher's one-tailed exact test was performed to generate a p-

836 value representing enrichment of the motif in peaks compared to matched length

837 random intergenic sequences.

\section{Genome-wide transcription profiling}

842 were performed on biofilms grown on the bottom of 6-well polystyrene plates for

84348 hours at $37^{\circ} \mathrm{C}$ and $200 \mathrm{rpm}$ as previously described for the determination of

844 biofilm biomass dry-weight (Nobile et al. 2012). The media used was Spider 1\%

845 glucose for all species, Spider for C. albicans and C. dubliniensis, and RPMI 1\%

846 glucose for C. albicans and C. tropicalis. Planktonic cultures for total RNA were

847 grown in the corresponding media by inoculating with cells from an overnight

$84830^{\circ} \mathrm{C}$ YPD culture to an $\mathrm{OD}_{600}$ of 0.05 . Cultures were then grown in flasks at $37^{\circ} \mathrm{C}$

849 with shaking at $225 \mathrm{rpm}$ until they reached an $\mathrm{OD}_{600}$ of 1.0. Biofilm and

850 planktonic cultures were harvested immediately by centrifugation at $3000 \mathrm{~g}$ for

851 three minutes and snap-frozen in liquid nitrogen. Total RNA was extracted from

852 the frozen pellets using the RiboPure-Yeast RNA kit (Ambion, AM1926) following

853 the manufacturer's recommendations. Transcription profiling was performed by

854 hybridization to custom-designed Agilent $8 * 15 \mathrm{k}$ oligonucleotide microarrays that

855 contain between 2 and 3 independent probes for each ORF (C. albicans AMADID

856 \#020166; C. dubliniensis, AMADID \#042592; C. tropicalis AMADID \#042593). 
857 cDNA synthesis, dye coupling, hybridization and microarray analysis was

858 performed as previously described (Nobile et al. 2012). Two biological replicates

859 were performed for each species in each condition using the wildtype strains.

860

861

\section{DATA DEPOSITION}

863 ChIP-Seq and microarray gene expression data has been deposited to the NCBI

864 Gene Expression Omnibus (GEO) repository under Superseries GSE160783.

865 


\section{ACKNOWLEDGMENTS}

867 We would like to thank Derek Sullivan and especially Joachim Morschhäuser for 868 providing strains, plasmids and advice, and Victor Hanson-Smith for help with 869 the ChIP-seq data analysis. This work was supported by a Human Frontier

870 Science Program grant to EM, a UC-MEXUS grant to EM, National Institutes of

871 Health (NIH) grants Ro1AIo83311 and Ro1AI049187 to ADJ, Ro1AIo73289 to

872 DRA, and R35GM124594 and R21AI125801 to CJN, by a Pew Biomedical Scholar

873 Award from the Pew Charitable Trusts to CJN, the Kamangar family in the form 874 of an endowed chair to CJN, CONACyT grant CB-2016-01 282511 to EM, and a 875 Welcome Trust Seed Award in Science to EM.

876

877

\section{CONTRIBUTIONS}

879 Conceptualization, EM and ADJ; Formal Analysis, EM and IN; Investigation, EM, 880 IN, SH, MG, KFM; Resources, EM, DRA, CJN, GB and ADJ; Writing, EM and 881 ADJ; Supervision, EM, DRA, CJN, GB and ADJ.

882

883

884 COMPETING INTERESTS

885 The authors declare that no competing interests exist. 


\section{FIGURE LEGENDS}

888 (Main figures are located at the end of the manuscript and include the 889 corresponding legend)

891 Figure 1. The biofilm transcription network in Candida albicans.

892 (A) The seven master transcription regulators identified in genetic screens 893 (Nobile et al. 2012; Fox et al. 2015), and the interactions among them as 894 determined by ChIP-seq and ChIP-qPCR. (B) Binding interactions (determined 895 by ChIP-seq) between the master regulators (red) and their target genes (black).

896 Many target genes are bound by more than one regulator. Note that genome-wide 897 binding data is not available for Flo8, and thus it is missing from the larger 898 network diagram. Figure 2. Diversity in biofilm formation across fungal species.

901 (A) Biofilm biomass dry weight was determined for different fungal species 902 grown on the bottoms of a polystyrene 6-well plates in Spider 1\% glucose medium 903 at $37^{\circ} \mathrm{C}$ for $48 \mathrm{hrs}$. The mean and standard deviation was calculated from five 904 replicates. Hashtags denote species that do not grow well at $37^{\circ} \mathrm{C}$ and for which 905 biofilms were grown at $30^{\circ} \mathrm{C}$. The cladogram to the left shows the phylogenetic 906 relationship of the species (Byrne and Wolfe 2005; Maguire et al. 2013). All 907 species analyzed belong to the CTG-Ser1 clade apart from C. glabrata and $S$. 908 cerevisiae. (B) Morphology of biofilms formed by five representative CTG clade 909 species visualized by CSLM. Biofilms were grown as described above, but on the 910 surface of silicone squares. Scale bars represent $50 \mu \mathrm{m}$. (C) Biofilm formation by 
911 Candida species in an in vivo rat catheter model (Andes et al. 2004). Biofilms

912 were grown for 24 hrs. and were visualized by scanning electron microscopy

913 (SEM). Two magnifications are shown in the lower and upper panel for each

914 species and the scale bars represent 100 and $20 \mu \mathrm{m}$, respectively.

916 Figure 3. Roles of orthologs of the seven C. albicans master

917 regulators in biofilm formation. (A) Phenotypic characterization of

918 biofilms formed by the gene deletion knockouts of orthologs of the seven master

919 C. albicans biofilm regulators. Images show biofilms grown on the bottoms of a

920 polystyrene 6-well plate in Spider $1 \%$ glucose medium at $37^{\circ} \mathrm{C}$ for $48 \mathrm{hrs}$. (B) Dry

921 weights of biofilms formed by the gene deletion mutants grown as described in

922 (A). The means and standard deviations were calculated from five replicates for

923 two independent gene deletion knockout isolates (KO-1 and $\mathrm{KO}-2)$. Asterisks

924 denote statistically significant different weights when compared to the

925 corresponding parental strain using a Student's two-tailed paired $t$ test $(P<$

926 0.05). (C) Summary diagram showing the conservation of the seven master

927 regulators in biofilm formation across the three most closely related species to $C$.

928 albicans. The data for C. albicans was obtained from (Nobile et al. 2012; Fox et

929 al. 2015), and that for C. parapsilosis from (Holland et al. 2014).

931 Figure 4. Connections between master regulators and target

932 genes are highly divergent across species. (A) Pair-wise comparison

933 of shared target genes for Ndt8o, Efg1 and Rob1 between species. Target genes 
934 were determined by ChIP-seq as detailed in the methods. The numbers represent

935 the percentage of overall target genes conserved between each pair of species

936 considering only genes that have orthologs in the two species. Note that Rob1 is

937 absent in C. parapsilosis (Maguire et al. 2013). (B) Venn diagrams depicting the

938 overlap of regulator-target gene connections across species, considering only

939 genes that have orthologs in all four species for Efg1 and Ndt8o and considering

940 genes that have orthologs in C. albicans, C. dubliniensi and C. tropicalis for Rob1.

941 Numbers in each section of the diagrams represent the percentage of master

942 regulator-target gene connections, with the total number of connections for each

943 regulator set at $100 \%$. Note that for Efg1 and Ndt8o the size of the color sections

944 do not correspond to the percentage. (C) Venn diagram depicting the overlap

945 between target genes of Efg1 and Ndt8o, considering only target gene orthologs

946 that are present in all four species. Numbers represent the gross number of

947 genes. The diagram indicates that, for genes that are targets of Efg1 and Ndt8o in

948 all species, most Efg1-target gene connections are also Ndt8o-target gene 949 connections, even though the target genes themselves are different across species.

952 Figure 5. Master regulators retain their DNA-binding specificity 953 while there is considerable variation in gene expression across

954 species. (A) Logos of the most enriched motif in the binding locations of the 955 different master regulators, determined by ChIP-seq across species. The two 956 circles to the right of each logo show whether the Efg1 (green circle) or Ndt80

957 (blue circle) previously known motifs are enriched in each set of regulator 
958 binding locations. (B) Pairwise comparison of transcription profiles under biofilm

959 forming conditions (a time point of 48 hours) of C. albicans against $C$.

960 dubliniensis and $C$. tropicalis. As a reference, the comparison between two

961 isolates of $C$. albicans is shown in the left panel. Biofilm specific expression

962 changes were calculated comparing gene expression between biofilm growing

963 conditions and planktonic cultures in the same media. Linear regressions are

964 shown in blue for each comparison.

965

966 Figure 6. Ndt80 ChIP-seq in a hybrid and rate of conservation

967 change of the different network components. (A) Genome-wide

968 comparison of $C$. albicans and C. dubliniensis Ndt8o binding in the hybrid strain.

969 Binding to both the C. albicans (dark blue filled dots) and the C. dubliniensis

970 (light blue empty dots) genomes is depicted. The maximal fold enrichment for

971 each upstream intergenic region in the genome is plotted as well as the linear

972 regression for each comparison. The left panel shows the C. albicans Ndt8o-C.

973 dubliniensis Ndt80 comparison while the right panel shows, as a reference, the

974 comparison of the two experimental replicates that are most dissimilar. (B)

975 Correlation between the master regulators required for biofilm formation, the

976 Efg1 and Ndt8o binding targets, and biofilm gene expression as a function of

977 evolutionary distance. Master regulator conservation is depicted as the

978 percentage of C. albicans regulators required for biofilm formation. Efg1 and

979 Ndt80 target conservation reflect the percentage of targets shared by the

980 different species pairs. Gene expression conservation represents the number of

981 genes whose expression changes at least $1.5 \log _{2}$ folds under biofilm forming 
982 conditions between each species pair. The C. parapsilosis gene expression data is

983 from (Holland et al. 2014). Linear regressions are shown in the corresponding

984 color. Evolutionary distance as substitutions per site was calculated from a

985 phylogenetic tree of these species, inferred from protein sequences of 73 highly

986 conserved genes (Lohse et al. 2013).

987

988 Supplementary Table 1. Biofilm formation of $C$. albicans and its three most

989 closely related species in different media. Biofilms were grown on silicone 990 squares at $37^{\circ} \mathrm{C}$ with shaking at $200 \mathrm{rpm}$. Biofilm formation was assessed by

991 CSLM after 48 hours of growth. Spider medium was prepared with 1\% nutrient 992 broth, $0.4 \%$ potassium phosphate and adjusted to $\mathrm{pH}$ 7.2. RPMI media used was 993 RMPI 1640 with $165 \mathrm{mM}$ MOPS and L-glutamine and without sodium

994 bicarbonate (Lonza, 04-525F). YNB media was prepared with $0.67 \%$ yeast 995 nitrogen base with ammonium sulfate.

997 Supplementary Table 2. Number of binding regions identified for each 998 regulator in the four Candida species studied. "NA", indicates experiments that 999 were not performed.

1000

1001 Supplementary Table 3. Master regulator binding to each other's upstream 1002 intergenic regions. Numbers represent the number of species in which binding 1003 was observed. The maximum possible number of species for which we have data 1004 is shown in parenthesis for each master regulator. 
1006 Supplementary Table 4. Strains used in this study.

1007

1008 Supplementary Figure 1. Time course of biofilm formation of C. albicans 1009 and its three most closely related Candida species. Biofilms were grown in Spider 1010 medium on silicone squares at $37^{\circ} \mathrm{C}$ with shaking at $200 \mathrm{rpm}$. Biofilm formation 1011 was assessed by CSLM after 30 minutes, 4, 8, 12, 24, 48 and 96 hours of biofilm 1012 growth. In each micrograph, the lower panel shows the lateral maximal intensity 1013 projection while the upper panel shows the top maximal intensity projection. 1014 Scale bars represent $50 \mu \mathrm{m}$.

1015

1016 Supplementary Figure 2. Biofilm formation in a microfluidic device by 1017 different CTG clade species was assayed as previously described (Gulati et al. 1018 2017). The medium used was Spider with $1 \%$ glucose and without mannitol. 1019 Assays were performed at $37^{\circ} \mathrm{C}$ and $30^{\circ} \mathrm{C}$ for 24 hours. The hashtag indicates the 1020 species that does not grow well at $37^{\circ} \mathrm{C}$ and for which biofilms were grown at $102130^{\circ} \mathrm{C}$

1022

1023 Supplementary Figure 3. Morphology of biofilms formed by the gene1024 deletion mutants of the biofilm regulators in C. dubliniensis and C. tropicalis 1025 visualized by CSLM. Biofilms were grown as described in the methods on the 1026 surface of silicone squares in Spider medium for C. dubliniensis and RPMI 1027 medium for $C$. tropicalis for $48 \mathrm{hrs}$ at $37^{\circ} \mathrm{C}$. Scale bars represent $50 \mu \mathrm{m}$. The $C$. 
1028 tropicalis ndt8o mutant as well as the flo8 mutants for both species were not 1029 analyzed by CSLM.

1030

1031 Supplementary Figure 4. Efg1 and Ndt8o target gene conservation between

1032 species when using different criteria to identify targets. This was performed 1033 similar to what was described previously (Nocedal et al. 2017). (A) Regulator 1034 gene target conservation when only using significant ChIP-seq signal over control 1035 in the upstream intergenic region as a criterion to identify gene targets. (B) 1036 Regulator gene target conservation when using ChIP-seq signal as in (A), but also 1037 filtering to include only those regions that also had a regulator binding motif in 1038 the upstream intergenic region. (C) Regulator gene target conservation when 1039 using ChIP-seq signal as in (A), but also two-fold gene expression change during 1040 biofilm growth conditions to identify target genes. (D) Regulator gene target 1041 conservation when using ChIP-seq signal, regulator binding motif presence and 1042 gene expression change during biofilm growth conditions to identify target genes.

1043 We did not perform gene expression profiling in C. parapsilosis and therefore 1044 gene expression change could not be used as a criterion for comparison with $C$. 1045 parapsilosis.

1046

1047 Supplementary Figure 5. Ndt8o binding throughout the genome in 1048 biofilms grown using different media. Biofilms were grown on the bottoms of 61049 well polystyrene plates for 48 hrs. as described in the methods. Binding was 1050 determined by ChIP-seq for biofilms grown in Spider 1\% glucose medium for the 1051 three species and compared to binding for biofilms grown in Spider medium for 
1052 C. albicans and C. dubliniensis, and RPMI medium for C. tropicalis. The 1053 maximum fold enrichment of each upstream intergenic region in the genome are 1054 plotted as well as the linear regression for each comparison. All correlations are 1055 highly significant (Pearson's correlation p-value $<<0.001$ ).

1056

1057 Supplementary Figure 6. Morphology of biofilms formed by the $C$. 1058 albicans-C. dubliniensis tetraploid hybrid (C. albicans $\mathrm{x}$ C. dubliniensis) 1059 visualized by CSLM. For comparison, the biofilms formed by C. albicans, C. 1060 dubliniensis and a C. albicans tetraploid (C. albicans x C. albicans) are also 1061 shown. Biofilms were grown as described in the methods on the surface of 1062 silicone squares in Spider medium for $48 \mathrm{hrs}$ at $37^{\circ} \mathrm{C}$. Scale bars represent $50 \mu \mathrm{m}$. 1063

1064 
1065

1066

1067

1068

1069

1070

1071

1072

1073

1074

1075

1076

1077

1078

1079

1080

1081

1082

1083

1084

1085

1086

1087

1088

1089

1090

1091

1092

1093

1094

1095

1096

1097

1098

1099

1100

1101

1102

1103

1104

1105

1106

1107

1108

\section{REFERENCES}

Andes, D., J. Nett, P. Oschel, R. Albrecht, K. Marchillo et al., 2004 Development and characterization of an in vivo central venous catheter Candida albicans biofilm model. Infect Immun 72: 6023-6031.

Araujo, D., M. Henriques and S. Silva, 2017 Portrait of Candida Species Biofilm Regulatory Network Genes. Trends Microbiol 25: 62-75.

Bailey, T. L., 2011 DREME: motif discovery in transcription factor ChIP-seq data. Bioinformatics 27: 1653-1659.

Basso, L. R., Jr., A. Bartiss, Y. Mao, C. E. Gast, P. S. Coelho et al., 2010 Transformation of Candida albicans with a synthetic hygromycin B resistance gene. Yeast 27: 1039-1048.

Blankenship, J. R., and A. P. Mitchell, 2006 How to build a biofilm: a fungal perspective. Curr Opin Microbiol 9: 588-594.

Brunet, T. D. P., and W. F. Doolittle, 2018 The generality of Constructive Neutral Evolution. Biology \& Philosophy 33: 2.

Butler, G., M. D. Rasmussen, M. F. Lin, M. A. Santos, S. Sakthikumar et al., 2009 Evolution of pathogenicity and sexual reproduction in eight Candida genomes. Nature 459: 657-662.

Byrne, K. P., and K. H. Wolfe, 2005 The Yeast Gene Order Browser: combining curated homology and syntenic context reveals gene fate in polyploid species. Genome Res 15: 1456-1461.

Calderone, R. A., and C. J. Clancy, 2012 Candida and Candidiasis, Second Edition. American Society of Microbiology.

Chen, Y., N. Negre, Q. Li, J. O. Mieczkowska, M. Slattery et al., 2012 Systematic evaluation of factors influencing ChIP-seq fidelity. Nat Methods 9: 609614.

Connolly, L. A., A. Riccombeni, Z. Grozer, L. M. Holland, D. B. Lynch et al., 2013 The APSES transcription factor Efg1 is a global regulator that controls morphogenesis and biofilm formation in Candida parapsilosis. Mol Microbiol 90: 36-53.

Ding, C., and G. Butler, 2007 Development of a gene knockout system in Candida parapsilosis reveals a conserved role for BCR1 in biofilm formation. Eukaryot Cell 6: 1310-1319.

Dominguez, E., R. Zarnowski, H. Sanchez, A. S. Covelli, W. M. Westler et al., 2018 Conservation and Divergence in the Candida Species Biofilm Matrix Mannan-Glucan Complex Structure, Function, and Genetic Control. mBio 9.

Donlan, R. M., 2001 Biofilm formation: a clinically relevant microbiological process. Clin Infect Dis 33: 1387-1392.

Fox, E. P., C. K. Bui, J. E. Nett, N. Hartooni, M. C. Mui et al., 2015 An expanded regulatory network temporally controls Candida albicans biofilm formation. Mol Microbiol 96: 1226-1239.

Gabaldon, T., M. A. Naranjo-Ortiz and M. Marcet-Houben, 2016 Evolutionary genomics of yeast pathogens in the Saccharomycotina. FEMS Yeast Res 16. 
Garcia-Sanchez, S., S. Aubert, I. Iraqui, G. Janbon, J. M. Ghigo et al., 2004 Candida albicans biofilms: a developmental state associated with specific and stable gene expression patterns. Eukaryot Cell 3: 536-545.

Gulati, M., C. L. Ennis, D. L. Rodriguez and C. J. Nobile, 2017 Visualization of Biofilm Formation in Candida albicans Using an Automated Microfluidic Device. J Vis Exp.

Hernday, A. D., S. M. Noble, Q. M. Mitrovich and A. D. Johnson, 2010 Genetics and molecular biology in Candida albicans. Methods Enzymol 470: 737758.

Hirakawa, M. P., D. A. Martinez, S. Sakthikumar, M. Anderson, A. Berlin et al., 2014 Genetic and phenotypic intra-species variation in Candida albicans. Genome Research.

Holland, L. M., M. S. Schroder, S. A. Turner, H. Taff, D. Andes et al., 2014 Comparative phenotypic analysis of the major fungal pathogens Candida parapsilosis and Candida albicans. PLoS Pathog 10: e1004365.

Homann, O. R., J. Dea, S. M. Noble and A. D. Johnson, 2009 A phenotypic profile of the Candida albicans regulatory network. PLoS Genet 5: e1000783.

Homann, O. R., and A. D. Johnson, 2010 MochiView: versatile software for genome browsing and DNA motif analysis. BMC Biol 8: 49.

Huang, M. Y., C. A. Woolford, G. May, C. J. McManus and A. P. Mitchell, 2019 Circuit diversification in a biofilm regulatory network. PLoS Pathog 15: e1007787.

Johnson, D. S., A. Mortazavi, R. M. Myers and B. Wold, 2007 Genome-wide mapping of in vivo protein-DNA interactions. Science 316: 1497-1502.

Kharchenko, P. V., M. Y. Tolstorukov and P. J. Park, 2008 Design and analysis of ChIP-seq experiments for DNA-binding proteins. Nat Biotechnol 26: 13511359.

Krassowski, T., A. Y. Coughlan, X. X. Shen, X. Zhou, J. Kominek et al., 2018 Evolutionary instability of CUG-Leu in the genetic code of budding yeasts. Nat Commun 9: 1887.

Kucharikova, S., H. Tournu, K. Lagrou, P. Van Dijck and H. Bujdakova, 2011 Detailed comparison of Candida albicans and Candida glabrata biofilms under different conditions and their susceptibility to caspofungin and anidulafungin. J Med Microbiol 60: 1261-1269.

Kullberg, B. J., and M. C. Arendrup, 2015 Invasive Candidiasis. N Engl J Med 373: $1445-1456$.

Kumari, A., S. Mankotia, B. Chaubey, M. Luthra and R. Singh, 2018 Role of biofilm morphology, matrix content and surface hydrophobicity in the biofilm-forming capacity of various Candida species. J Med Microbiol 67: 889-892.

Kurtzman, C. P., J. W. Fell and T. Boekhout, 2011 The Yeasts.

Langmead, B., and S. L. Salzberg, 2012 Fast gapped-read alignment with Bowtie 2. Nat Methods 9: 357-359.

Lassak, T., E. Schneider, M. Bussmann, D. Kurtz, J. R. Manak et al., 2011 Target specificity of the Candida albicans Efg1 regulator. Molecular Microbiology 82: 602-618. 
1156

Li, H., B. Handsaker, A. Wysoker, T. Fennell, J. Ruan et al., 2009 The Sequence Alignment/Map format and SAMtools. Bioinformatics 25: 2078-2079.

Lohse, M. B., I. V. Ene, V. B. Craik, A. D. Hernday, E. Mancera et al., 2016 Systematic Genetic Screen for Transcriptional Regulators of the Candida albicans White-Opaque Switch. Genetics 203: 1679-1692.

Lohse, M. B., M. Gulati, A. D. Johnson and C. J. Nobile, 2018 Development and regulation of single- and multi-species Candida albicans biofilms. Nat Rev Microbiol 16: 19-31.

Lohse, M. B., M. Gulati, A. Valle Arevalo, A. Fishburn, A. D. Johnson et al., 2017 Assessment and Optimizations of Candida albicans In Vitro Biofilm Assays. Antimicrob Agents Chemother 61.

Lohse, M. B., A. D. Hernday, P. M. Fordyce, L. Noiman, T. R. Sorrells et al., 2013 Identification and characterization of a previously undescribed family of sequence-specific DNA-binding domains. Proc Natl Acad Sci U S A 110: $7660-7665$.

Lohse, M. B., and A. D. Johnson, 2016 Identification and Characterization of Wor4, a New Transcriptional Regulator of White-Opaque Switching. G3 (Bethesda) 6: 721-729.

Lynch, D. P., 1994 Oral candidiasis. History, classification, and clinical presentation. Oral Surg Oral Med Oral Pathol 78: 189-193.

Lynch, M., 2007 The origins of genome architecture. Sinauer Associates Sunderland, MA.

Maguire, S. L., S. S. Oheigeartaigh, K. P. Byrne, M. S. Schroder, P. O'Gaora et al., 2013 Comparative Genome Analysis and Gene Finding in Candida Species Using CGOB. Mol Biol Evol 30: 1281-1291.

Mancera, E., C. Frazer, A. M. Porman, S. Ruiz-Castro, A. D. Johnson et al., 2019 Genetic Modification of Closely Related Candida Species. Front Microbiol 10: 357 .

Mishra, P. K., M. Baum and J. Carbon, 2007 Centromere size and position in Candida albicans are evolutionarily conserved independent of DNA sequence heterogeneity. Mol Genet Genomics 278: 455-465.

Moran, G. P., D. C. Coleman and D. J. Sullivan, 2012 Candida albicans versus Candida dubliniensis: Why Is C. albicans More Pathogenic? Int J Microbiol 2012: 205921.

Nobile, C. J., E. P. Fox, J. E. Nett, T. R. Sorrells, Q. M. Mitrovich et al., 2012 A recently evolved transcriptional network controls biofilm development in Candida albicans. Cell 148: 126-138.

Nobile, C. J., and A. D. Johnson, 2015 Candida albicans Biofilms and Human Disease. Annu Rev Microbiol 69: 71-92.

Noble, S. M., and A. D. Johnson, 2005 Strains and strategies for large-scale gene deletion studies of the diploid human fungal pathogen Candida albicans. Eukaryot Cell 4: 298-309.

Nocedal, I., E. Mancera and A. D. Johnson, 2017 Gene regulatory network plasticity predates a switch in function of a conserved transcription regulator. Elife 6. 
1201

1202

1203

1204

1205

1206

1207

1208

1209

1210

1211

1212

1213

1214

1215

1216

1217

1218

1219

1220

1221

1222

1223

1224

1225

1226

1227

1228

1229

1230

1231

1232

1233

1234
Porman, A. M., K. Alby, M. P. Hirakawa and R. J. Bennett, 2011 Discovery of a phenotypic switch regulating sexual mating in the opportunistic fungal pathogen Candida tropicalis. Proc Natl Acad Sci U S A 108: 21158-21163.

Pujol, C., K. J. Daniels, S. R. Lockhart, T. Srikantha, J. B. Radke et al., 2004 The closely related species Candida albicans and Candida dubliniensis can mate. Eukaryot Cell 3: 1015-1027.

Pujol, C., K. J. Daniels and D. R. Soll, 2015 Comparison of Switching and Biofilm Formation between MTL-Homozygous Strains of Candida albicans and Candida dubliniensis. Eukaryot Cell 14: 1186-1202.

Ramage, G., K. Vande Walle, B. L. Wickes and J. L. Lopez-Ribot, 2001 Biofilm formation by Candida dubliniensis. J Clin Microbiol 39: 3234-3240.

Reuss, O., A. Vik, R. Kolter and J. Morschhauser, 2004 The SAT1 flipper, an optimized tool for gene disruption in Candida albicans. Gene 341: 119-127.

Richard, M. L., C. J. Nobile, V. M. Bruno and A. P. Mitchell, 2005 Candida albicans biofilm-defective mutants. Eukaryot Cell 4: 1493-1502.

Romo, J. A., and C. A. Kumamoto, 2020 On Commensalism of Candida. J Fungi (Basel) 6.

Silva, S., M. Negri, M. Henriques, R. Oliveira, D. W. Williams et al., 2011 Adherence and biofilm formation of non-Candida albicans Candida species. Trends Microbiol 19: 241-247.

Sorrells, T. R., and A. D. Johnson, 2015 Making sense of transcription networks. Cell 161: 714-723.

Stoltzfus, A., 1999 On the possibility of constructive neutral evolution. J Mol Evol 49: 169-181.

Turner, S. A., and G. Butler, 2014 The Candida pathogenic species complex. Cold Spring Harb Perspect Med 4: a019778.

Wagner, A., 2014 Arrival of the fittest: How nature innovates. Current.

Wilson, M. D., N. L. Barbosa-Morais, D. Schmidt, C. M. Conboy, L. Vanes et al., 2008 Species-Specific Transcription in Mice Carrying Human Chromosome 21. Science 322: 434-438.

Zhang, Y., T. Liu, C. A. Meyer, J. Eeckhoute, D. S. Johnson et al., 2008 Modelbased analysis of ChIP-Seq (MACS). Genome Biol 9: R137. 
Figure 1

A

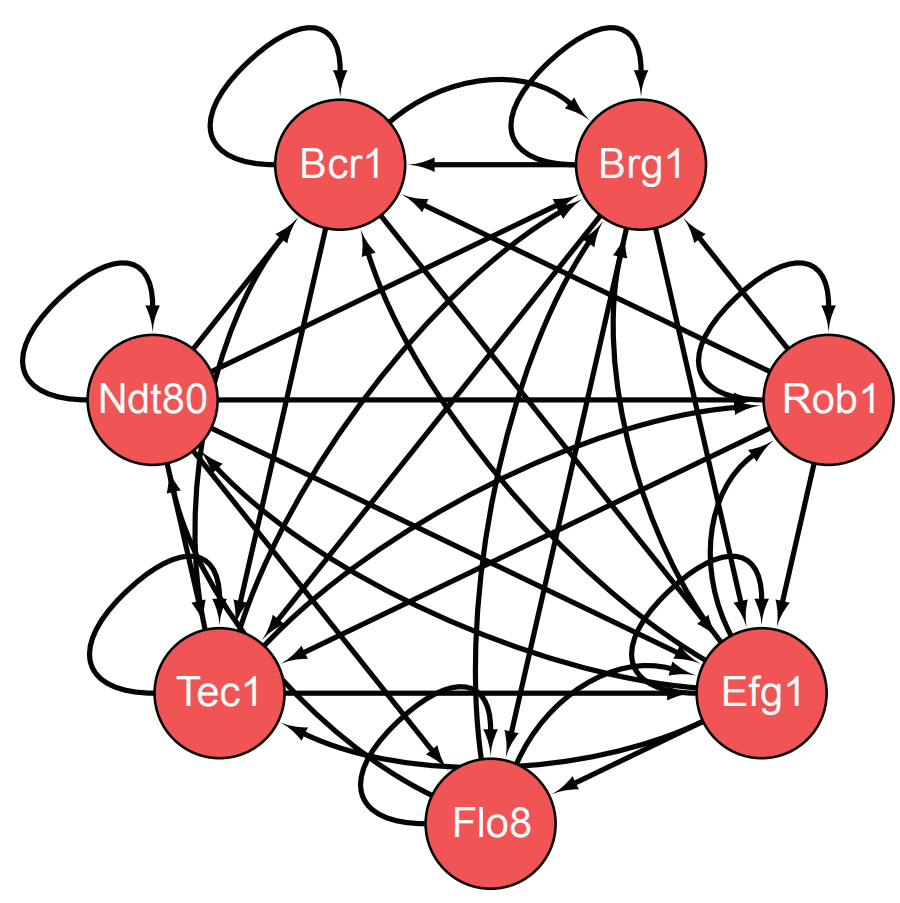

B

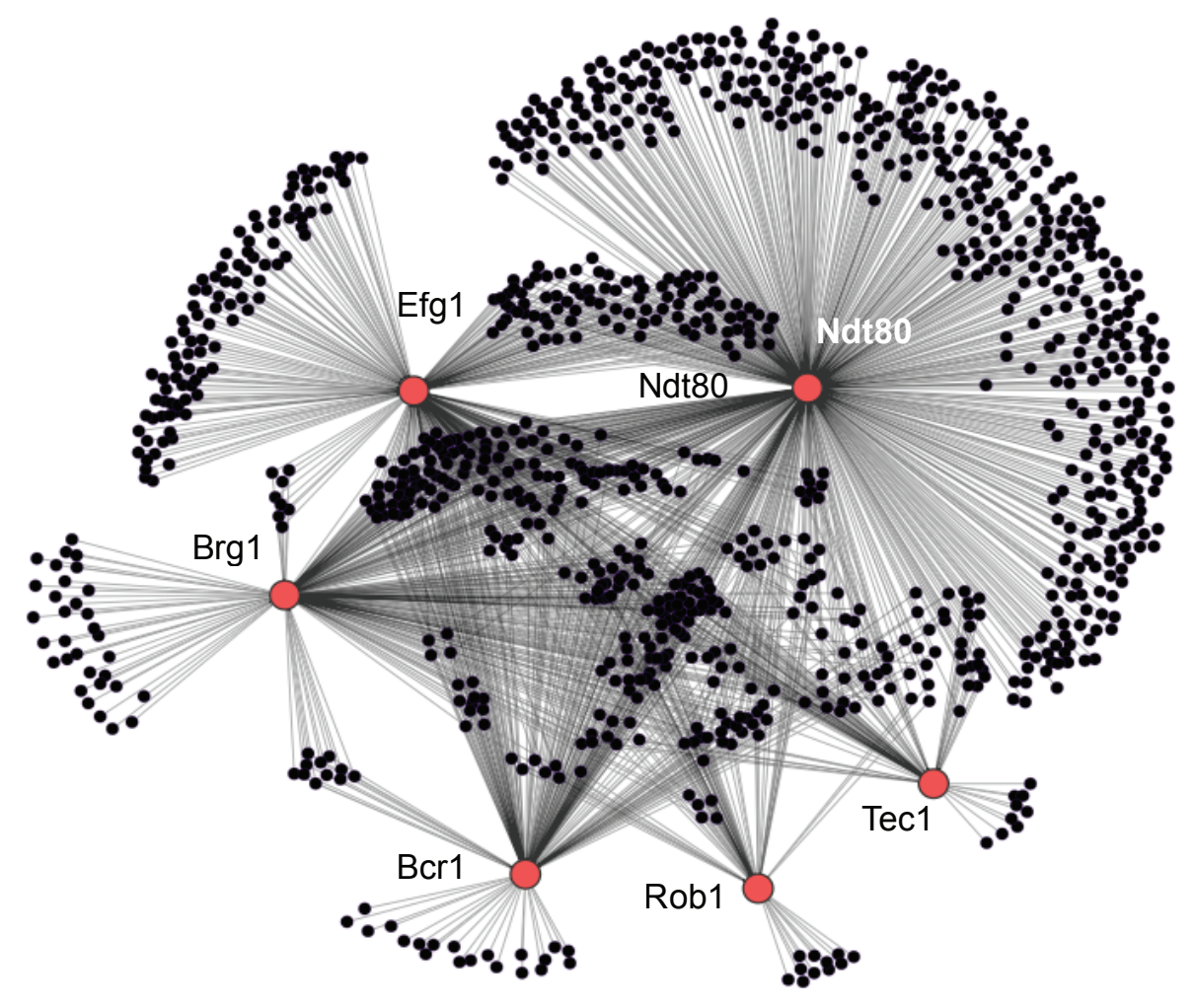


bioRxiv preprint doi: https://doi.org/10.1101/2020.11.08.373514; this version posted November 10, 2020. The copyright holder for this preprint (which was not certified by peer review) is the author/funder. All rights reserved. No reuse allowed without permission.

Figure 1. The biofilm transcription network in Candida albicans. (A) The seven master transcription regulators identified in genetic screens (Nobile et al. 2012; Fox et al. 2015), and the interactions among them as determined by ChIP-seq and ChIP-qPCR. (B) Binding interactions (determined by ChIP-seq) between the master regulators (red) and their target genes (black). Many target genes are bound by more than one regulator. Note that genome-wide binding data is not available for Flo8, and thus it is missing from the larger network diagram. 


\section{Figure 2}

A

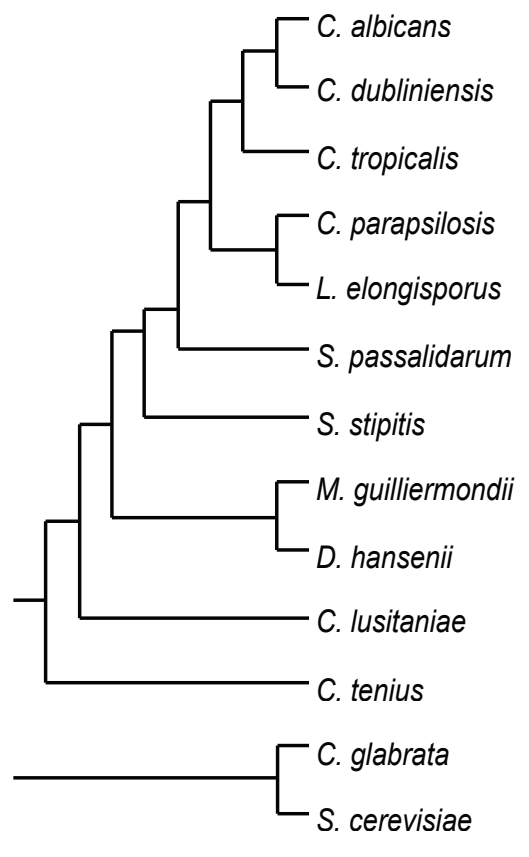

Biomass Dry Weight (mg)

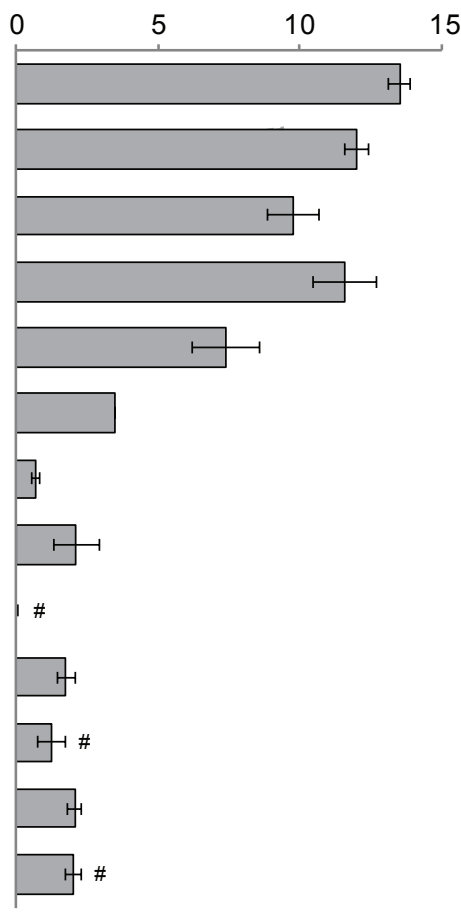

B Top view Side view
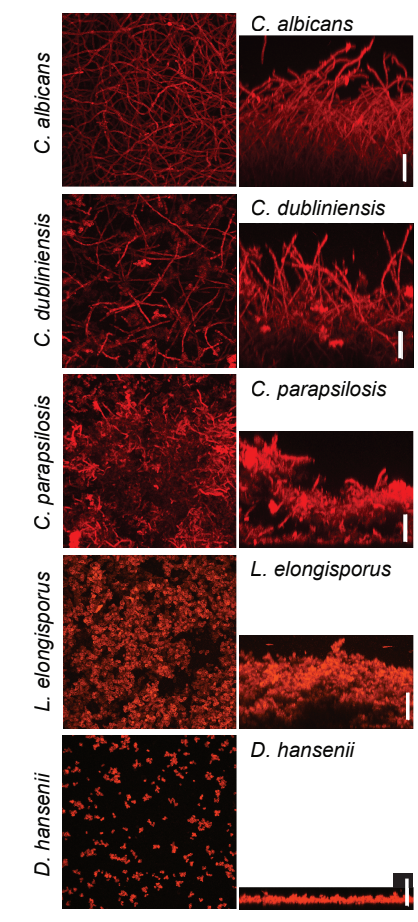

\section{C}

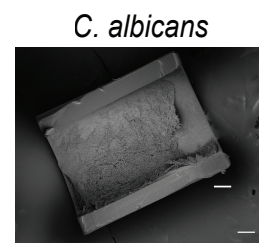

C. dubliniensis

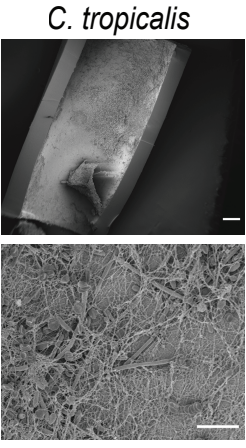

C. parapsilosis
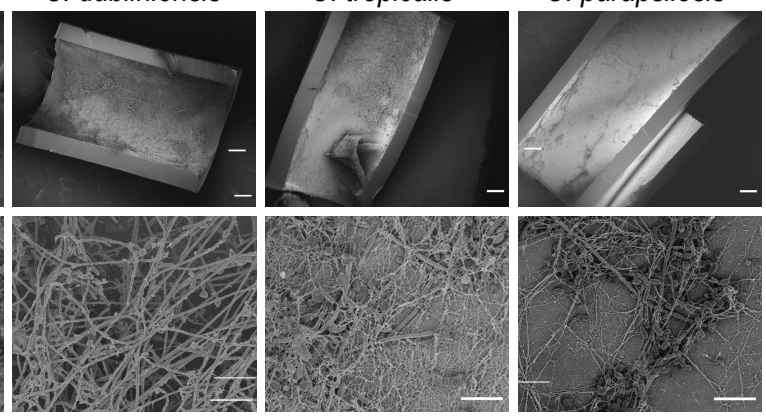

Figure 2. Diversity in biofilm formation across fungal species. (A) Biofilm biomass dry weight was determined for different fungal species grown on the bottoms of a polystyrene 6 -well plates in Spider $1 \%$ glucose medium at $37^{\circ} \mathrm{C}$ for $48 \mathrm{hrs}$. The mean and standard deviation was calculated from five replicates. Hashtags denote species that do not grow well at $37^{\circ} \mathrm{C}$ and for which biofilms were grown at $30^{\circ} \mathrm{C}$. The cladogram to the left shows the phylogenetic relationship of the species (Byrne and Wolfe 2005; Maguire et al. 2013). All species analyzed belong to the CTG-Ser1 clade apart from C. glabrata and S. cerevisiae. (B) Morphology of biofilms formed by five representative CTG clade species visualized by CSLM. Biofilms were grown as described above, but on the surface of silicone squares. Scale bars represent $50 \mu \mathrm{m}$. (C) Biofilm formation by Candida species in an in vivo rat catheter model (Andes et al. 2004). Biofilms were grown for $24 \mathrm{hrs}$. and were visualized by scanning electron microscopy (SEM). Two magnifications are shown in the lower and upper panel for each species and the scale bars represent 100 and $20 \mu \mathrm{m}$, respectively. 


\section{Figure 3}

A

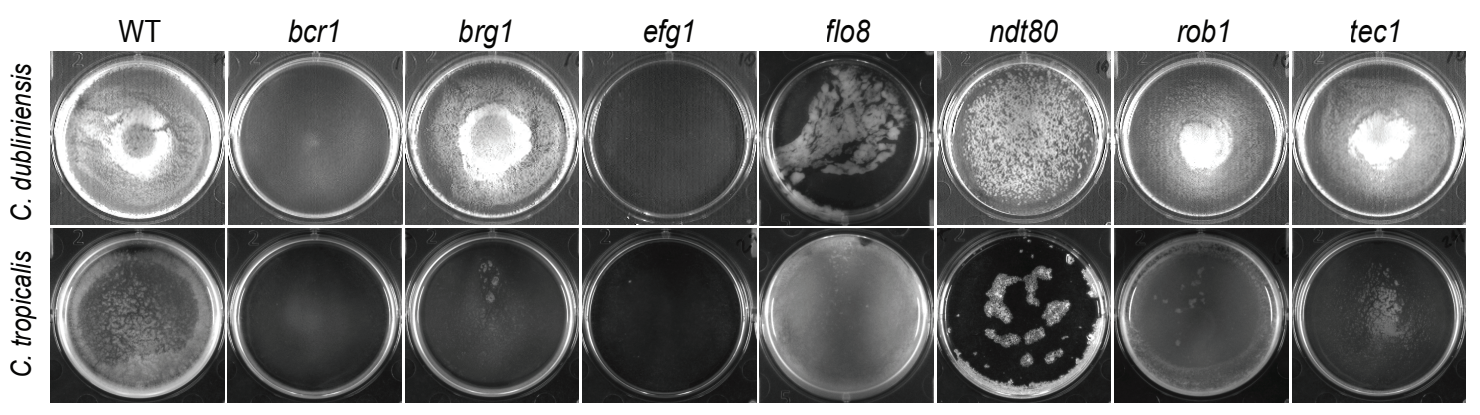

B

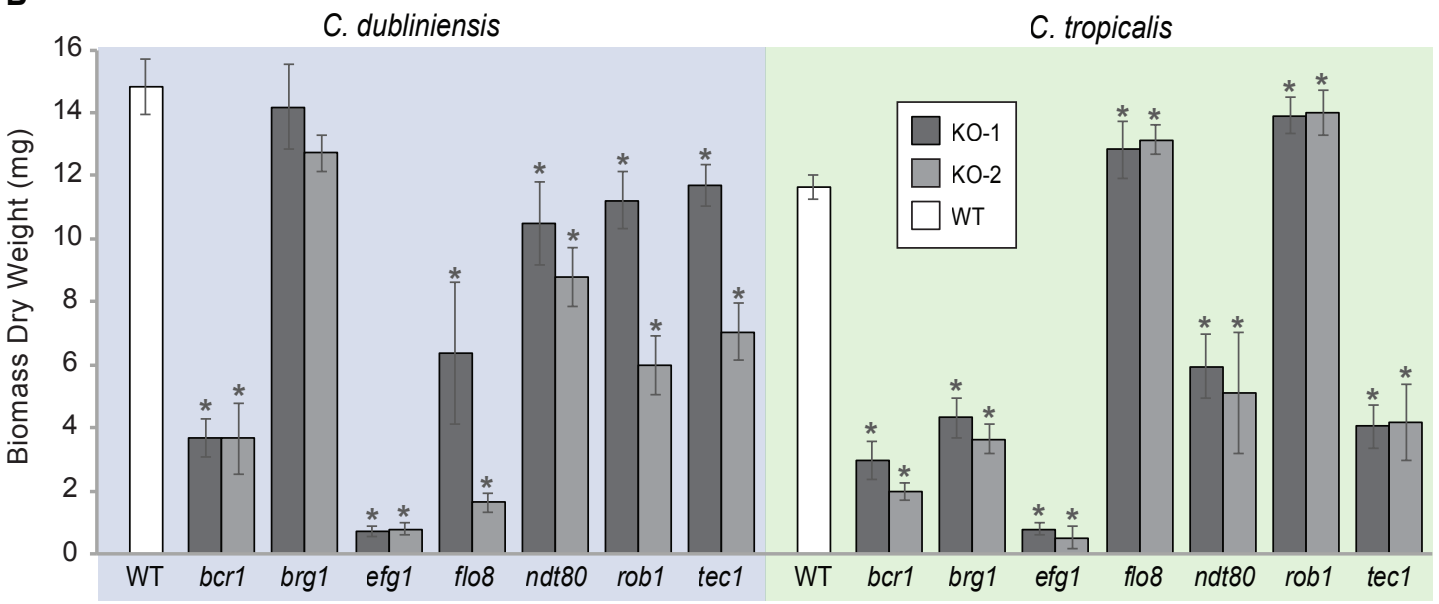

C

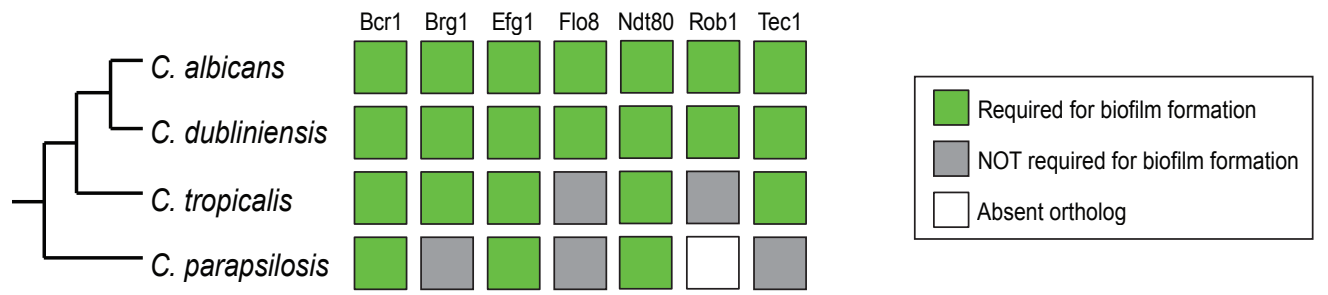

Figure 3. Roles of orthologs of the seven C. albicans master regulators in biofilm formation. (A) Phenotypic characterization of biofilms formed by the gene deletion knockouts of orthologs of the seven master $\mathrm{C}$. albicans biofilm regulators. Images show biofilms grown on the bottoms of a polystyrene 6-well plate in Spider $1 \%$ glucose medium at $37^{\circ} \mathrm{C}$ for $48 \mathrm{hrs}$. (B) Dry weights of biofilms formed by the gene deletion mutants grown as described in (A). The means and standard deviations were calculated from five replicates for two independent gene deletion knockout isolates (KO-1 and KO-2). Asterisks denote statistically significant different weights when compared to the corresponding parental strain using a Student's two-tailed paired t test $(P<0.05)$. (C) Summary diagram showing the conservation of the seven master regulators in biofilm formation across the three most closely related species to C. albicans. The data for $\mathrm{C}$. albicans was obtained from (Nobile et al. 2012; Fox et al. 2015), and that for C. parapsilosis from (Holland et al. 2014). 


\section{Figure 4}

A

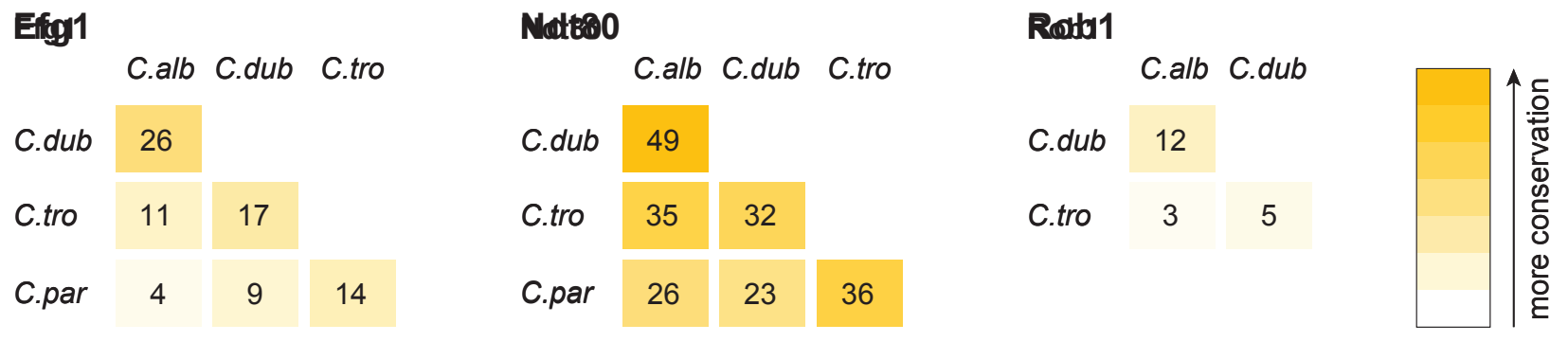

\section{B}

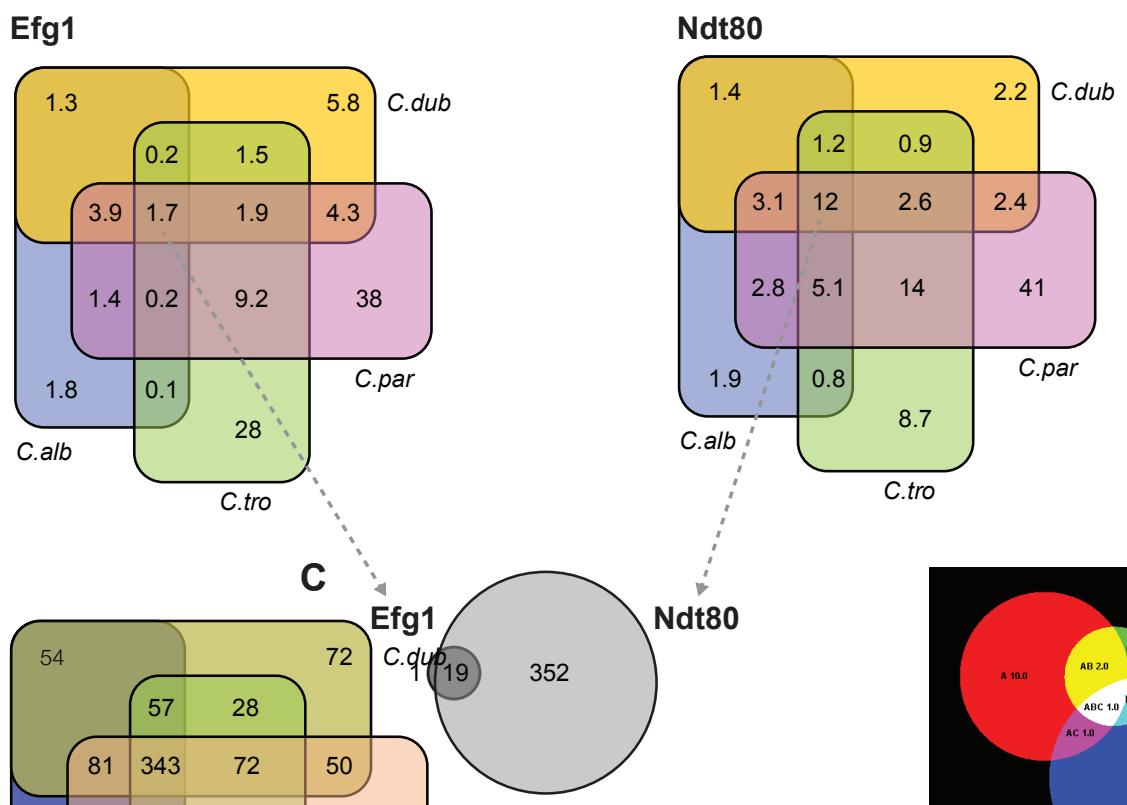

Rob1

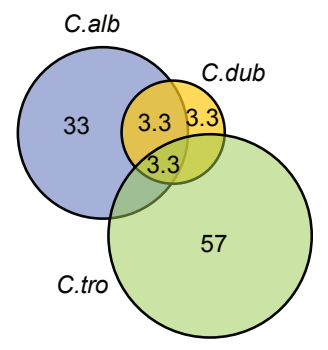

Figure 4. Connections between master regulators and target genes are highly divergent across species. (A) Pair-wise comparison of shared target genes for Ndt80, Efg1 and Rob1 between species. Target genes were determined by ChIP-seq as detailed in the methods. The numbers represent the percentage of overall target genes conserved between each pair of species considering only genes that have orthologs in the two species. Note that Rob1 is absent in C. parapsilosis (Maguire et al. 2013). (B) Venn diagrams depicting the overlap of regulator-target gene connections across species, considering only genes that have orthologs in all four species for $\mathrm{Efg} 1$ and $\mathrm{Ndt} 80$ and considering genes that have orthologs in C. albicans, C. dubliniensi and C. tropicalis for Rob1. Numbers in each section of the diagrams represent the percentage of master regulator-target gene connections, with the total number of connections for each regulator set at $100 \%$. Note that for Efg1 and Ndt80 the size of the color sections do not correspond to the percentage. (C) Venn diagram depicting the overlap between target genes of Efg1 and Ndt80, considering only target gene orthologs that are present in all four species. Numbers represent the gross number of genes. The diagram indicates that, for genes that are targets of Efg1 and Ndt80 in all species, most Efg1-target gene connections are also Ndt80-target gene connections, even though the target genes themselves are different across species. 


\section{Figure 5}

A

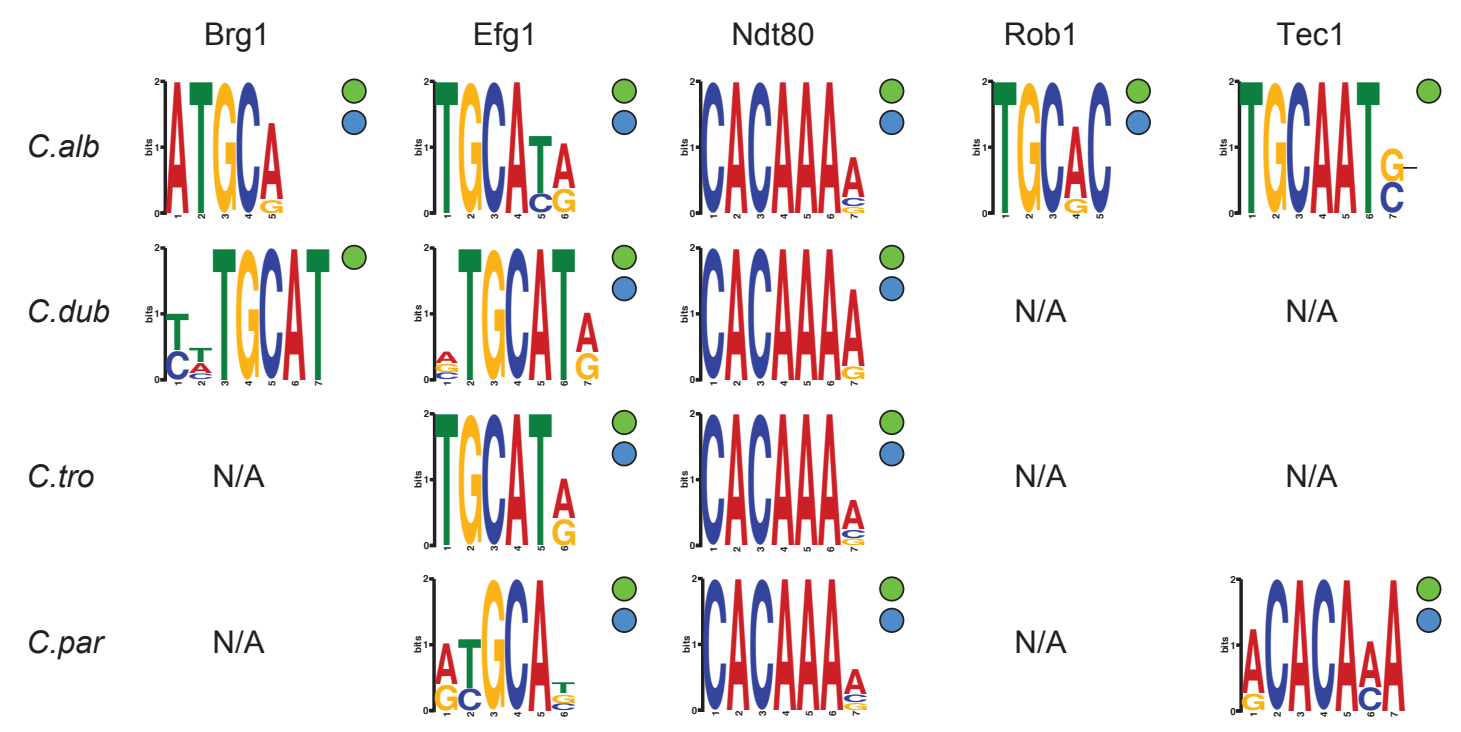

B
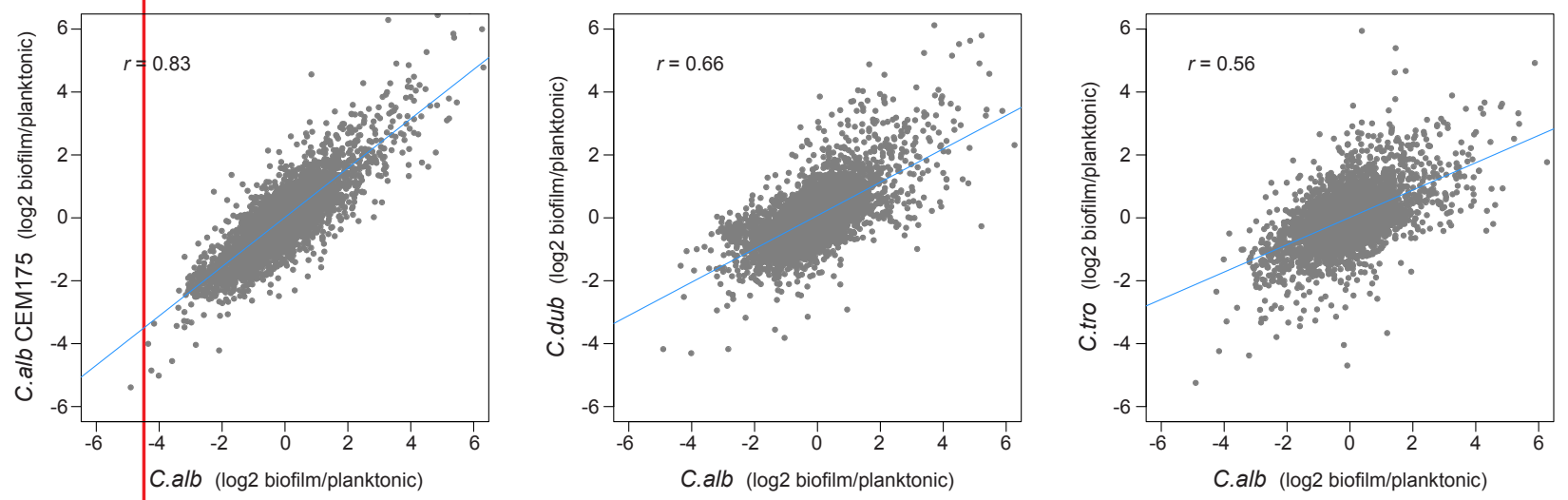

Figure 5. Master regulators retain their DNA-binding specificity while there is considerable variation in gene expression across species. (A) Logos of the most enriched motif in the binding locations of the different master regulators, determined by ChIP-seq across species. The two circles to the right of each logo show whether the Efg1 (green circle) or Ndt80 (blue circle) previously known motifs are enriched in each set of regulator binding locations. (B) Pairwise comparison of transcription profiles under biofilm forming conditions (a time point of 48 hours) of C. albicans against C. dubliniensis and $\mathrm{C}$. tropicalis. As a reference, the comparison between two isolates of $\mathrm{C}$. albicans is shown in the left panel. Biofilm specific expression changes were calculated comparing gene expression between biofilm growing conditions and planktonic cultures in the same media. Linear regressions are shown in blue for each comparison. 


\section{Figure 6}

A

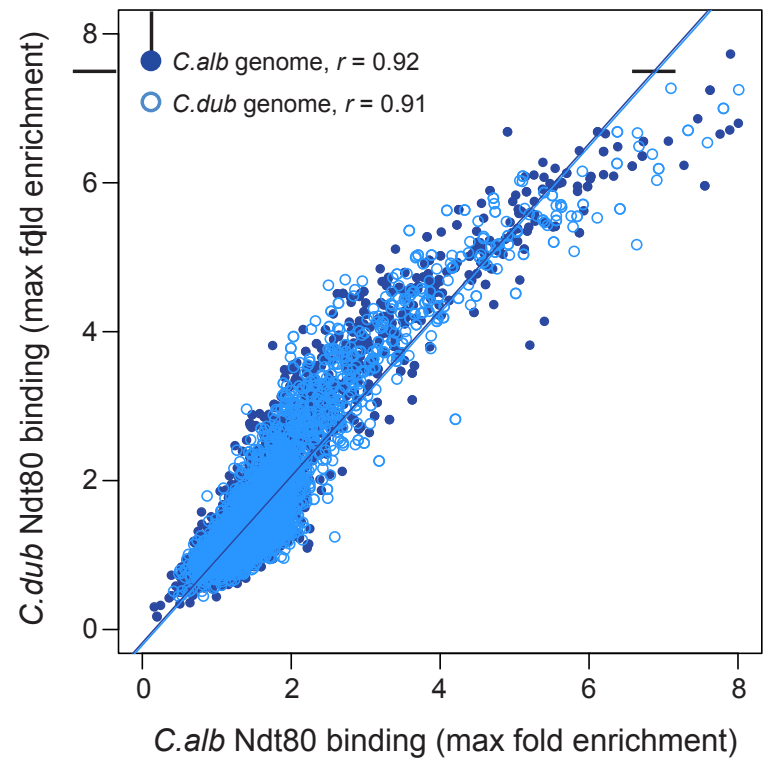

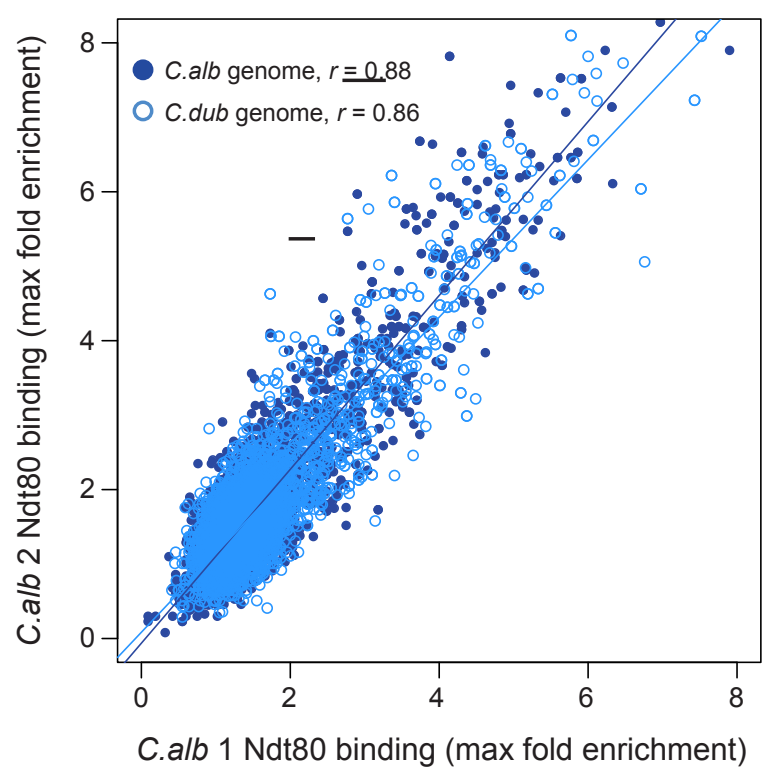

C.alb 1 Ndt80 binding (max fold enrichment)

B

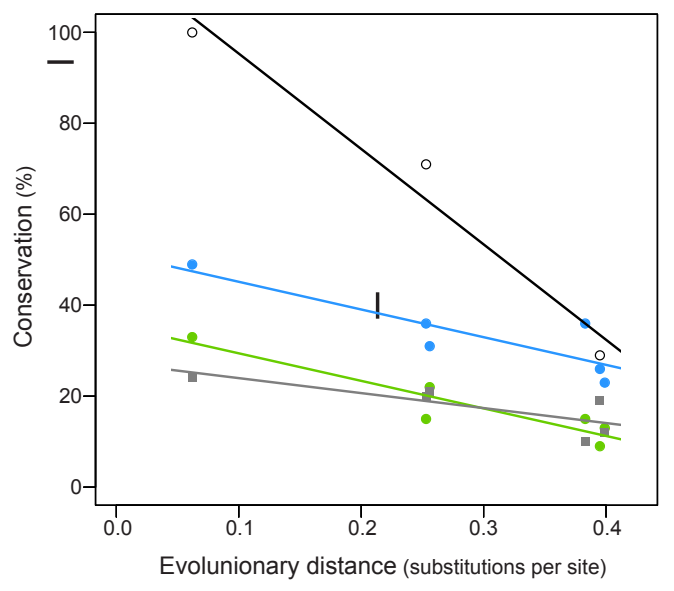

Figure 6. Ndt80 ChIP-seq in a hybrid and rate of conservation change of the different network components. (A) Genomewide comparison of $\mathrm{C}$. albicans and $\mathrm{C}$. dubliniensis $\mathrm{Ndt80}$ binding in the hybrid strain. Binding to both the $\mathrm{C}$. albicans (dark blue filled dots) and the $\mathrm{C}$. dubliniensis (light blue empty dots) genomes is depicted. The maximal fold enrichment for each upstream intergenic region in the genome is plotted as well as the linear regression for each comparison. The left panel shows the $\mathrm{C}$. albicans Ndt80-C. dubliniensis Ndt80 comparison while the right panel shows, as a reference, the comparison of the two experimental replicates that are most dissimilar. (B) Correlation between the master regulators required for biofilm formation, the Efg1 and Ndt80 binding targets, and biofilm gene expression as a function of evolutionary distance. Master regulator conservation is depicted as the percentage of $\mathrm{C}$. albicans regulators required for biofilm formation. Efg1 and Ndt80 target conservation reflect the percentage of targets shared by the different species pairs. Gene expression conservation represents the number of genes whose expression changes at least 1.5 log2 folds under biofilm forming conditions between each species pair. The C. parapsilosis gene expression data is from (Holland et al. 2014). Linear regressions are shown in the corresponding color. Evolutionary distance as substitutions per site was calculated from a phylogenetic tree of these species, inferred from protein sequences of 73 highly conserved genes (Lohse et al. 2013). 